TITLE:

\title{
Master equation for the Unruh- DeWitt detector and the universal relaxation time in de Sitter space
}

\section{AUTHOR(S):}

Fukuma, Masafumi; Sugishita, Sotaro; Sakatani, Yuho

\section{CITATION:}

Fukuma, Masafumi ...[et al]. Master equation for the Unruh-DeWitt detector and the universal relaxation time in de Sitter space. Physical Review D 2014, 89(6): 064024.

ISSUE DATE:

2014-03-10

URL:

http://hdl.handle.net/2433/187100

RIGHT:

(C) 2014 American Physical Society 
PHYSICAL REVIEW D 89, 064024 (2014)

\title{
Master equation for the Unruh-DeWitt detector and the universal relaxation time in de Sitter space
}

\author{
Masafumi Fukuma, and Sotaro Sugishita \\ Department of Physics, Kyoto University, Kyoto 606-8502, Japan \\ Yuho Sakatani \\ Maskawa Institute for Science and Culture, Kyoto Sangyo University, Kyoto 603-8555, Japan
}

(Received 1 October 2013; published 10 March 2014)

\begin{abstract}
We derive the master equation that completely determines the time evolution of the density matrix of the Unruh-DeWitt detector in an arbitrary background geometry. We apply the equation to reveal a nonequilibrium thermodynamic character of de Sitter space. This generalizes an earlier study on the thermodynamic property of the Bunch-Davies vacuum that an Unruh-DeWitt detector staying in the Poincaré patch and interacting with a scalar field in the Bunch-Davies vacuum behaves as if it is in a thermal bath of finite temperature. In this paper, instead of the Bunch-Davies vacuum, we consider a class of initial states of scalar field, for which the detector behaves as if it is in a medium that is not in thermodynamic equilibrium and that undergoes a relaxation to the equilibrium corresponding to the BunchDavies vacuum. We give a prescription for calculating the relaxation times of the nonequilibrium processes. We particularly show that, when the initial state of the scalar field is the instantaneous ground state at a finite past, the relaxation time is always given by a universal value of half the curvature radius of de Sitter space. We expect that the relaxation time gives a nonequilibrium thermodynamic quantity intrinsic to de Sitter space.
\end{abstract}

\section{INTRODUCTION}

The concept of particles is known to depend on observers. Even in the Poincaré-invariant Minkowski vacuum, an observer with constant acceleration sees a thermal particle spectrum [1]. The Unruh-DeWitt detector $[1,2]$ was introduced as a tool of thought experiment to give an intuitive understanding of such thermal character of spacetime (see also [3,4], and references therein). This is a detector weakly interacting with a matter quantum field in a certain vacuum state, and one can study the thermal character of spacetime through the density distribution of the detector.

Various spacetimes have been examined with the Unruh-DeWitt detector, including de Sitter space. Although a free scalar field in de Sitter space has a large family of de Sitter-invariant vacua (called the $\alpha$ vacua) $[5,6]$, the Bunch-Davies vacuum (or the Euclidean vacuum) [7] is regarded as the most natural vacuum, because this satisfies the Hadamard condition. It is actually only the Bunch-Davies vacuum which exhibits a thermal property [3,8-11]; if one places a detector at $\boldsymbol{x}=\mathbf{0}$ in the Poincaré patch,

\footnotetext{
*ukuma@gauge.scphys.kyoto-u.ac.jp sotaro@gauge.scphys.kyoto-u.ac.jp yuho@cc.kyoto-su.ac.jp
}

$$
\mathrm{d} s^{2}=\ell^{2} \frac{-\mathrm{d} \eta^{2}+\mathrm{d} x^{2}}{\eta^{2}} \quad(-\infty<\eta<0),
$$

where the scalar field is initially in the Bunch-Davies vacuum, then the density distribution of the detector evolves through the interaction with the scalar field and eventually reaches the Gibbs distribution of temperature $T=1 / 2 \pi \ell$ ( $\ell$ being the curvature radius of de Sitter space), irrespectively of the initial form of the density distribution [see Fig. 1(a)]. This implies that the detector behaves as if it is in a thermal bath of temperature $T=$ $1 / 2 \pi \ell$ when it is placed in the Bunch-Davies vacuum.

The specialty of the Bunch-Davies vacuum may be understood as follows. We first notice that there is no global timelike Killing vector in the Poincaré patch, and thus the Hamiltonian of the scalar field has an explicit time dependence. This implies that one cannot define a time-independent ground state and can only introduce the instantaneous ground state $\left|0_{\eta}\right\rangle$ at each instant $\eta$. As is investigated in detail in Ref. [12], the BunchDavies vacuum $|\mathrm{BD}\rangle$ can be characterized as the ground state at the infinite past: $|\mathrm{BD}\rangle=\lim _{\eta \rightarrow-\infty}\left|0_{\eta}\right\rangle$. Thus, one may regard the Bunch-Davies vacuum as a medium (surrounding the detector) which already undergoes a sufficiently long time evolution to reach a thermodynamic equilibrium state.

If instead the scalar field is initially in a certain class of states, the Unruh-DeWitt detector may behave as if it is 


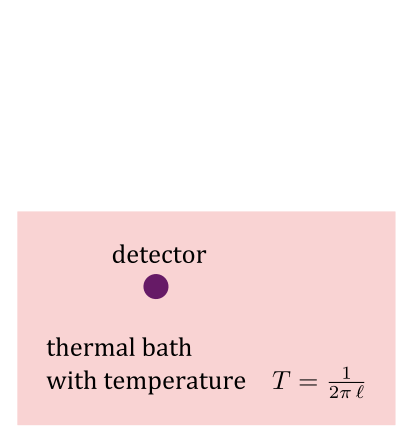

(a)

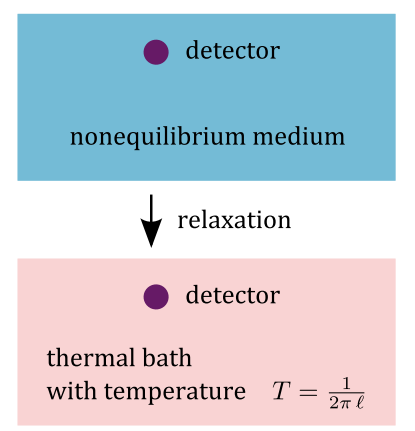

(b)
FIG. 1 (color online). The Unruh-DeWitt detector in the Poincaré patch. The scalar field is initially in (a) the BunchDavies vacuum or (b) a class of states deviated slightly from the Bunch-Davies vacuum.

surrounded by a medium that is not in thermodynamic equilibrium and that undergoes a relaxation to the equilibrium corresponding to the Bunch-Davies vacuum [see Fig. 1(b)]. It should be possible to investigate the relaxation process of the surrounding medium by observing the time evolution of the density distribution of the detector. This analysis may give useful information on the nonequilibrium thermodynamic character intrinsic to de Sitter space.

The main purpose of this paper is to develop a machinery for describing such nonequilibrium dynamics and to calculate the relaxation times of the surrounding media. For this, we first develop a general framework to treat an Unruh-DeWitt detector in arbitrary background geometry and derive the master equation which completely determines a finite time evolution of the density matrix of the detector. We then apply this framework to a detector in de Sitter space. We show that if the initial state of the scalar field is chosen such that its Wightman function has the same short distance behavior as that of the Bunch-Davies vacuum, then the density distribution of any detector placed there exhibits a relaxation to the Gibbs distribution, with a relaxation time proportional to $\ell$ (measured in the proper time of the detector). In particular, if we take the initial state as the instantaneous ground state at a finite past (say, at $\eta_{0}$ ), the relaxation time is always given by a universal value $\ell / 2$.

In order to avoid possible confusions, we here stress that there can be two kinds of relaxation times. The first is the relaxation time that may exist even when the detector is placed in a thermal bath [see Fig. 1(a)]. This is the period of time it takes for the detector to reach the Gibbs distribution from a given initial density distribution. This kind of relaxation time can be neglected if one considers an ideal detector which can get adjusted to its environment instantaneously. Another kind of relaxation time, in which we are interested, is the period of time it takes for the nonequilibrium environment to reach a thermodynamic equilibrium state [see Fig. 1(b)]. This relaxation time should not depend on details of the detector or on the form of interaction between the detector and the scalar field and is related to the nonequilibrium dynamics intrinsic to de Sitter space.

This paper is organized as follows. In Sec. II, adopting the method of the projection operator [13], we first derive the master equation which describes the time evolution of the density matrix of the detector. Then, after justifying a Markovian approximation, we derive a simplified form of the master equation which enables us to study the relaxation behavior of the density distribution analytically. In Sec. III, we apply the framework to an Unruh-DeWitt detector in the Poincaré patch of de Sitter space. We consider a situation where the initial state of the scalar field is chosen such that its Wightman function has the same short distance behavior as that of the Bunch-Davies vacuum. We compute the transition rate matrix of the density distribution of the detector and show that the density distribution exhibits the expected relaxation to the equilibrium corresponding to the Bunch-Davies vacuum with the relaxation time of the form $\ell / \alpha$, where the constant $\alpha$ is determined by the asymptotic form of the change of the Wightman function from that of the BunchDavies vacuum. In Sec. IV, we consider a particular case where the initial state of the scalar field is the instantaneous ground state $\left|0_{\eta_{0}}\right\rangle$ at a finite past $\eta=\eta_{0}$ and show that the relaxation time is always given by a universal value $\ell / 2$, irrespectively of the value of $\eta_{0}$ or the form of interaction between the detector and the scalar field. Section V is devoted to discussions and conclusion. We collect miscellaneous formulas in the Appendixes.

\section{MASTER EQUATION FOR THE DENSITY MATRIX OF AN UNRUH-DEWITT DETECTOR}

\section{A. Setup}

We consider an Unruh-DeWitt detector in $d$-dimensional spacetime with background metric $\mathrm{d} s^{2}=g_{\mu \nu}(x) \mathrm{d} x^{\mu} \mathrm{d} x^{\nu}$ $(\mu, \nu=0,1, \ldots, d-1)$, the detector interacting with a scalar field $\phi(x)$ of mass $m$. We assume that the detector has a sufficiently large mass so that it can be treated as moving along a classical trajectory ${ }^{1} x^{\mu}(\tau)=(t(\tau), \boldsymbol{x}(\tau))$, where $\tau$ is a proper time of the trajectory (see Fig. 2).

For a quantum mechanical description of the system, we introduce the Hilbert space $\mathcal{H}^{\text {tot }}$, which is the tensor product of those of the detector and the field:

$$
\mathcal{H}^{\text {tot }}=\mathcal{H}^{\mathrm{d}} \otimes \mathcal{H}^{\phi}
$$

The total Hamiltonian then takes the following form in the Schrödinger picture:

\footnotetext{
${ }^{1}$ We also write the trajectory as $x^{\mu}(\tau)=(t, \boldsymbol{x}(t))$ by using the functional relation $t=t(\tau)$ or $\tau=\tau(t)$ with $\mathrm{d} t / \mathrm{d} \tau>0$.
} 


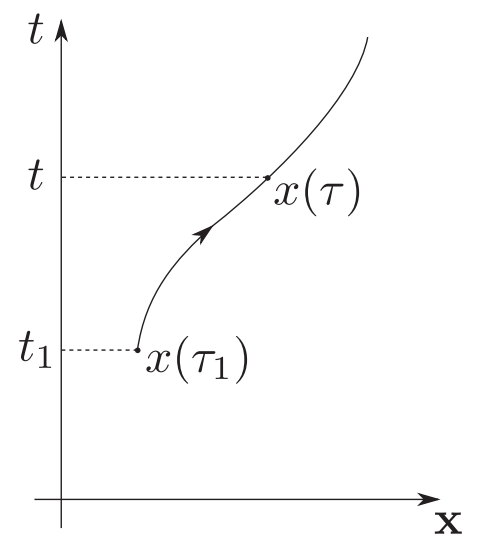

FIG. 2. The trajectory of an Unruh-DeWitt detector, which starts interacting with a scalar field $\phi(x)$ at time $t_{1}$. The time coordinate $t$ will be denoted by $\eta$ when it is the conformal time in the Poincaré patch.

$$
H^{\mathrm{tot}}(t)=H^{\mathrm{d}} \frac{\mathrm{d} \tau(t)}{\mathrm{d} t} \otimes 1+1 \otimes H^{\phi}(t)+V(t) .
$$

Here, $H^{\mathrm{d}}$ is the Hamiltonian of the detector associated with its proper time, and we assume that $H^{\mathrm{d}}$ does not depend on $\tau$ (or on $t$ ), denoting its eigenstates and eigenvalues by $|m\rangle$ and $E_{m}$, respectively: $H^{\mathrm{d}}|m\rangle=E_{m}|m\rangle . H^{\phi}(t)$ is the Hamiltonian of the free scalar field and may depend on time explicitly through an explicit time dependence of the metric. $V(t)$ stands for the interaction between the detector and the field, and we assume that it suddenly starts at time $t_{1}$ in the form of a monopole interaction, ${ }^{2}$

$$
V(t)=\lambda \mu \frac{\mathrm{d} \tau(t)}{\mathrm{d} t} \otimes \phi(\boldsymbol{x}(t)) \theta\left(t-t_{1}\right)
$$

Here, $\lambda$ is a dimensionless coupling constant, $\boldsymbol{x}(t)$ represents the position of the detector at time $t$ (see footnote 1), and $\mu$ is an operator acting on $\mathcal{H}^{\mathrm{d}}$, which we again assume to be time independent. The time evolution of the total density matrix $\rho^{\text {tot }}(t)$ is given by

$$
\rho^{\mathrm{tot}}(t)=U^{\mathrm{tot}}\left(t, t^{\prime}\right) \rho^{\mathrm{tot}}\left(t^{\prime}\right)\left[U^{\mathrm{tot}}\left(t, t^{\prime}\right)\right]^{-1},
$$

where $U^{\text {tot }}\left(t, t^{\prime}\right)$ is the time evolution operator in the Schrödinger picture,

$$
U^{\mathrm{tot}}\left(t, t^{\prime}\right) \equiv \mathrm{T} \exp \left(-\mathrm{i} \int_{t^{\prime}}^{t} \mathrm{~d} t^{\prime} H^{\mathrm{tot}}\left(t^{\prime}\right)\right)
$$

\footnotetext{
${ }^{2}$ Recall that this is in the Schrödinger picture. We later shall take an average over the startup time $t_{1}$, since one usually needs a certain period of time to specify the initial density distribution of the detector.
}

We consider a situation where one can measure only observables associated with the detector, such as the matrix elements $\mu_{m n}=\langle m|\mu| n\rangle$. Then the maximum information one can get from the system is the reduced density matrix $\rho(t)$, which is defined as the partial trace of the total density matrix $\rho^{\text {tot }}(t)$ over $\mathcal{H}^{\phi}$ :

$$
\rho(t) \equiv \operatorname{Tr}_{\phi} \rho^{\mathrm{tot}}(t)
$$

The time evolution of $\rho(t)$ should be uniquely determined once one specifies the form of interaction and the initial condition for $\rho^{\text {tot }}(t)$. Since there had been no interaction between the detector and the field before time $t_{1}$, we may let the total density matrix take the following factorized form at $t_{1}$ :

$$
\rho^{\mathrm{tot}}\left(t_{1}\right) \equiv \rho^{\mathrm{d}}\left(t_{1}\right) \otimes \rho^{\phi}\left(t_{1}\right)=\rho\left(t_{1}\right) \otimes \rho^{\phi}\left(t_{1}\right) .
$$

The latter equality can be easily seen by noting that $\rho\left(t_{1}\right)=\operatorname{Tr}_{\phi} \rho^{\mathrm{tot}}\left(t_{1}\right)=\rho^{\mathrm{d}}\left(t_{1}\right)$.

\section{B. Master equation}

The time evolution of $\rho(t)$ can be best analyzed if we go over to the interaction picture by decomposing the total Hamiltonian to

$$
\begin{gathered}
H^{\mathrm{tot}}(t)=H_{0}^{\mathrm{tot}}(t)+V(t), \\
H_{0}^{\mathrm{tot}}(t) \equiv H^{\mathrm{d}} \frac{\mathrm{d} \tau(t)}{\mathrm{d} t} \otimes 1+1 \otimes H^{\phi}(t)
\end{gathered}
$$

and treat $V(t)$ as a perturbation. The time evolution operator $U^{\text {tot }}\left(t, t_{1}\right)$ is then decomposed to the unperturbed and perturbed parts as

$$
U^{\text {tot }}\left(t, t_{1}\right)=U_{0}^{\mathrm{tot}}\left(t, t_{1}\right) U_{I}^{\mathrm{tot}}\left(t, t_{1}\right)
$$

with

$$
\begin{aligned}
U_{0}^{\mathrm{tot}}\left(t, t_{1}\right) & \equiv \mathrm{T} \exp \left(-\mathrm{i} \int_{t_{1}}^{t} \mathrm{~d} t^{\prime} H_{0}^{\mathrm{tot}}\left(t^{\prime}\right)\right) \\
& =e^{-\mathrm{i} H^{\mathrm{d}} \cdot\left(\tau-\tau_{1}\right)} \otimes \mathrm{T} e^{-\mathrm{i} \int_{t_{1}}^{t} \mathrm{~d} t^{\prime} H^{\phi}\left(t^{\prime}\right)}, \\
U_{I}^{\mathrm{tot}}\left(t, t_{1}\right) & \equiv \mathrm{T} \exp \left(-\mathrm{i} \int_{t_{1}}^{t} \mathrm{~d} t^{\prime} V_{I}\left(t^{\prime}\right)\right) .
\end{aligned}
$$

Here, $V_{I}(t)$ is defined by 


$$
\begin{aligned}
V_{I}(t) & \equiv\left[U_{0}^{\text {tot }}\left(t, t_{1}\right)\right]^{-1} V(t) U_{0}^{\text {tot }}\left(t, t_{1}\right) \\
& =\lambda \frac{\mathrm{d} \tau}{\mathrm{d} t} \mu_{I}(\tau) \otimes \phi_{I}(x(\tau)) \theta\left(t-t_{1}\right),
\end{aligned}
$$

where $\mu_{I}(\tau)$ is given by

$$
\begin{aligned}
\mu_{I}(\tau) & \equiv e^{\mathrm{i} H^{\mathrm{d}} \cdot\left(\tau-\tau_{1}\right)} \mu e^{-\mathrm{i} H^{\mathrm{d}} \cdot\left(\tau-\tau_{1}\right)} \\
& =\sum_{m, n} e^{\mathrm{i}\left(E_{m}-E_{n}\right)\left(\tau-\tau_{1}\right)} \mu_{m n}|m\rangle\langle n|,
\end{aligned}
$$

and the operator

$$
\begin{aligned}
\phi_{I}(x(\tau)) & \equiv \phi_{I}(t, \boldsymbol{x}(t)) \\
& =\left[\mathrm{T} e^{-\mathrm{i} \int_{t_{1}}^{t} \mathrm{~d} t^{\prime} H^{\phi}\left(t^{\prime}\right)}\right]^{-1} \phi(\boldsymbol{x}(t)) \mathrm{T} e^{-\mathrm{i} \int_{t_{1}}^{t} \mathrm{~d} t^{\prime} H^{\phi}\left(t^{\prime}\right)}
\end{aligned}
$$

satisfies the free Klein-Gordon equation associated with the metric $\mathrm{d} s^{2}=g_{\mu \nu}(x) \mathrm{d} x^{\mu} \mathrm{d} x^{\nu}$. Accordingly, the density matrix in the interaction picture is given by

$$
\begin{aligned}
\rho_{I}^{\mathrm{tot}}(t) & \equiv\left[U_{0}^{\mathrm{tot}}\left(t, t_{1}\right)\right]^{-1} \rho^{\mathrm{tot}}(t) U_{0}^{\mathrm{tot}}\left(t, t_{1}\right) \\
& =U_{I}^{\mathrm{tot}}\left(t, t_{1}\right) \rho^{\mathrm{tot}}\left(t_{1}\right)\left[U_{I}^{\mathrm{tot}}\left(t, t_{1}\right)\right]^{-1}
\end{aligned}
$$

and satisfies the von Neumann equation of the form

$$
\frac{\mathrm{d}}{\mathrm{d} t} \rho_{I}^{\mathrm{tot}}(t)=-\mathrm{i}\left[V_{I}(t), \rho_{I}^{\mathrm{tot}}(t)\right] \equiv-\mathrm{i} \operatorname{ad}_{V_{I}(t)} \rho_{I}^{\mathrm{tot}}(t)
$$

This von Neumann equation can be rewritten to an equation involving only $\rho_{I}(t) \equiv \operatorname{Tr}_{\phi} \rho_{I}^{\text {tot }}(t)$ by adopting the projection operator method (see, e.g., [13]). We first introduce a linear operator $\mathcal{P}$ : End $\mathcal{H}^{\text {tot }} \rightarrow$ End $\mathcal{H}^{\text {tot }}$, which acts linearly on elements belonging to End $\mathcal{H}^{\text {tot }}$ (the set of linear operators acting on $\mathcal{H}^{\text {tot }}$ ) and has the form

$$
\mathcal{P}: O \mapsto \mathcal{P} O \equiv\left(\operatorname{Tr}_{\phi} O\right) \otimes X^{\phi} \quad\left(O \in \text { End } \mathcal{H}^{\text {tot }}\right)
$$

Here, $X^{\phi} \in$ End $\mathcal{H}^{\phi}$ can be any operator acting on $\mathcal{H}^{\phi}$ as long as it satisfies

$$
\operatorname{Tr}_{\phi} X^{\phi}=1
$$

and does not depend on time. From (20) one can easily see that $\mathcal{P}$ is a projection operator, $\mathcal{P}^{2}=\mathcal{P}$. For $O=\rho_{I}^{\text {tot }}(t)$, we obtain

$$
\mathcal{P} \rho_{I}^{\text {tot }}(t)=\left(\operatorname{Tr}_{\phi} \rho_{I}^{\text {tot }}(t)\right) \otimes X^{\phi}=\rho_{I}(t) \otimes X^{\phi} .
$$

We further introduce $\mathcal{Q} \equiv 1-\mathcal{P}$, which is also a projection operator, $\mathcal{Q}^{2}=\mathcal{Q}$, and satisfies $\mathcal{P} \mathcal{Q}=0=\mathcal{Q P}$.

Using the fact that operator $X^{\phi}$ can be chosen arbitrarily without changing the time evolution of $\rho_{I}(t)$ (as far as it satisfies the aforementioned conditions), we here set $X^{\phi} \equiv \rho^{\phi}\left(t_{1}\right)=\rho_{I}^{\phi}\left(t_{1}\right)$. This certainly satisfies the condition (20), and due to the initial condition (8) the following equations hold:

$$
\mathcal{P} \rho_{I}^{\text {tot }}\left(t_{1}\right)=\rho_{I}^{\text {tot }}\left(t_{1}\right), \quad \mathcal{Q} \rho_{I}^{\text {tot }}\left(t_{1}\right)=0 .
$$

Then, if the one-point function of scalar field vanishes (as we assume hereafter),

$$
\operatorname{Tr}_{\phi}\left(\phi_{I}(x(\tau)) \rho_{I}^{\phi}\left(t_{1}\right)\right)=0,
$$

we obtain the equation

$$
\begin{aligned}
\frac{\mathrm{d} \rho_{I}(t)}{\mathrm{d} t} \otimes \rho_{I}^{\phi}\left(t_{1}\right)= & -\mathcal{P a d}_{V_{I}(t)} \int_{t_{1}}^{t} \mathrm{~d} t^{\prime} \mathrm{T} e^{-\mathrm{i} \int_{t^{\prime}}^{t} \mathrm{~d}^{\prime \prime} \operatorname{Qad}_{V_{I}\left(t^{\prime \prime}\right)}} \\
& \times \operatorname{ad}_{V_{I}\left(t^{\prime}\right)}\left(\rho_{I}\left(t^{\prime}\right) \otimes \rho_{I}^{\phi}\left(t_{1}\right)\right) .
\end{aligned}
$$

We give a proof of Eq. (24) in Appendix A. This is the master equation which with the initial condition (8) and the assumption (23) completely determines the time evolution of the reduced density matrix $\rho_{I}(t)$.

\section{Approximation of the master equation}

We expand the right-hand side of (24) to the second order in perturbation to obtain

$$
\begin{aligned}
\frac{\mathrm{d} \rho_{I}(t)}{\mathrm{d} t}= & -\operatorname{Tr}_{\phi}\left(\operatorname{ad}_{V_{I}(t)} \int_{t_{1}}^{t} \mathrm{~d} t^{\prime} \operatorname{ad}_{V_{I}\left(t^{\prime}\right)}\left(\rho_{I}\left(t^{\prime}\right) \otimes \rho_{I}^{\phi}\left(t_{1}\right)\right)\right) \\
& +\mathcal{O}\left(\lambda^{3}\right) .
\end{aligned}
$$

This can be further rewritten in terms of the proper time $\tau$ to the following form [denoting $\rho(t(\tau))$ by $\rho(\tau)$ and derivatives with respect to $\tau$ by dots]:

$$
\begin{aligned}
\dot{\rho}_{I}(\tau) & =-\lambda^{2} \int_{\tau_{1}}^{\tau} \mathrm{d} \tau^{\prime} \operatorname{Tr}_{\phi}\left[\mu_{I}(\tau) \otimes \phi_{I}(x(\tau)),\left[\mu_{I}\left(\tau^{\prime}\right) \otimes \phi_{I}\left(x\left(\tau^{\prime}\right)\right), \rho_{I}\left(\tau^{\prime}\right) \otimes \rho_{I}^{\phi}\left(\tau_{1}\right)\right]\right]+\mathcal{O}\left(\lambda^{3}\right) \\
& =\lambda^{2} \int_{\tau_{1}}^{\tau} \mathrm{d} \tau^{\prime}\left(\left[\mu_{I}(\tau), \rho_{I}\left(\tau^{\prime}\right) \mu_{I}\left(\tau^{\prime}\right)\right] G_{X}^{+}\left(x\left(\tau^{\prime}\right), x(\tau)\right)-\left[\mu_{I}(\tau), \mu_{I}\left(\tau^{\prime}\right) \rho_{I}\left(\tau^{\prime}\right)\right] G_{X}^{+}\left(x(\tau), x\left(\tau^{\prime}\right)\right)\right)+\mathcal{O}\left(\lambda^{3}\right),
\end{aligned}
$$




\section{MASTER EQUATION FOR THE UNRUH-DEWITT DETECTOR ...}

PHYSICAL REVIEW D 89, 064024 (2014)

where

$$
G_{X}^{+}\left(x, x^{\prime}\right) \equiv \operatorname{Tr}_{\phi}\left(\phi_{I}(x) \phi_{I}\left(x^{\prime}\right) \rho_{I}^{\phi}\left(\tau_{1}\right)\right)
$$

is the Wightman function of the free scalar field with respect to the density matrix $X^{\phi}=\rho_{I}^{\phi}\left(\tau_{1}\right)$.

Since the reduced density matrix in the interaction picture, $\rho_{I}(\tau)$, is related to that in the Schrödinger picture, $\rho(\tau)$, as

$$
\rho_{I}(\tau)=e^{\mathrm{i} H^{\mathrm{d}} \cdot\left(\tau-\tau_{1}\right)} \rho(\tau) e^{-\mathrm{i} H^{\mathrm{d}} \cdot\left(\tau-\tau_{1}\right)},
$$

we can rewrite (26) to the following form in the Schrödinger picture:

$$
\begin{aligned}
\dot{\rho}(\tau) & +\mathrm{i}\left[H^{\mathrm{d}}, \rho(\tau)\right] \\
= & \lambda^{2} \int_{\tau_{1}}^{\tau} \mathrm{d} \tau^{\prime}\left[\mu e^{-\mathrm{i} H^{\mathrm{d}} \cdot\left(\tau-\tau^{\prime}\right)} \rho\left(\tau^{\prime}\right) \mu e^{\mathrm{i} H^{\mathrm{d}} \cdot\left(\tau-\tau^{\prime}\right)} G_{X}^{+}\left(x\left(\tau^{\prime}\right), x(\tau)\right)\right. \\
& +e^{-\mathrm{i} H^{\mathrm{d} \cdot}\left(\tau-\tau^{\prime}\right)} \mu \rho\left(\tau^{\prime}\right) \mu e^{\mathrm{i} H^{\mathrm{d}} \cdot\left(\tau-\tau^{\prime}\right)} G_{X}^{+}\left(x(\tau), x\left(\tau^{\prime}\right)\right) \\
& -e^{-\mathrm{i} H^{\mathrm{d}} \cdot\left(\tau-\tau^{\prime}\right)} \rho\left(\tau^{\prime}\right) \mu e^{\mathrm{i} H^{\mathrm{d}} \cdot\left(\tau-\tau^{\prime}\right)} \mu G_{X}^{+}\left(x\left(\tau^{\prime}\right), x(\tau)\right) \\
& \left.-\mu e^{-\mathrm{i} H^{\mathrm{d}} \cdot\left(\tau-\tau^{\prime}\right)} \mu \rho\left(\tau^{\prime}\right) e^{\mathrm{i} H^{\mathrm{d}} \cdot\left(\tau-\tau^{\prime}\right)} G_{X}^{+}\left(x(\tau), x\left(\tau^{\prime}\right)\right)\right]+\mathcal{O}\left(\lambda^{3}\right) .
\end{aligned}
$$

The integro-differential equation (29) can be further simplified as follows. Since the Wightman function $G_{X}^{+}\left(x(\tau), x\left(\tau^{\prime}\right)\right)$ in the integral is singular at $\tau^{\prime}=\tau$ and decreases exponentially for large separations of $\tau$ and $\tau^{\prime}{ }^{3}$, the main contributions to the integral should come only from the region $\tau^{\prime} \sim \tau$. This implies that the memory effect in the equation is highly suppressed, and thus we may replace $\rho_{k l}\left(\tau^{\prime}\right)$ in the integral by its boundary value $\rho_{k l}(\tau)$ to a good accuracy, assuming that $\rho\left(\tau^{\prime}\right)$ slowly changes. Equation (29) can thus be rewritten (in terms of matrix elements) as

$$
\begin{aligned}
& \dot{\rho}_{m n}(\tau)+\mathrm{i}\left(E_{m}-E_{n}\right) \rho_{m n}(\tau) \\
& \simeq \lambda^{2} \sum_{k, l} \int_{\tau_{1}}^{\tau} \mathrm{d} \tau^{\prime}\left[e^{-\mathrm{i}\left(E_{n}-E_{k}\right)\left(\tau^{\prime}-\tau\right)} \mu_{m k} \mu_{l n} \rho_{k l}(\tau) G_{X}^{+}\left(x\left(\tau^{\prime}\right), x(\tau)\right)\right. \\
& \quad+e^{-\mathrm{i}\left(E_{m}-E_{l}\right)\left(\tau-\tau^{\prime}\right)} \mu_{m k} \mu_{l n} \rho_{k l}(\tau) G_{X}^{+}\left(x(\tau), x\left(\tau^{\prime}\right)\right) \\
& \quad-e^{-\mathrm{i}\left(E_{l}-E_{m}\right)\left(\tau^{\prime}-\tau\right)} \mu_{k l} \mu_{l n} \rho_{m k}(\tau) G_{X}^{+}\left(x\left(\tau^{\prime}\right), x(\tau)\right) \\
& \left.\quad-e^{-\mathrm{i}\left(E_{k}-E_{n}\right)\left(\tau-\tau^{\prime}\right)} \mu_{m k} \mu_{k l} \rho_{l n}(\tau) G_{X}^{+}\left(x(\tau), x\left(\tau^{\prime}\right)\right)\right]
\end{aligned}
$$

\footnotetext{
${ }^{3}$ We see in Appendix D that for a scalar field in de Sitter space the Wightman function certainly exhibits this property if the mass is large enough. In general, there can be a case where the Wightman function has a long tail and one needs to take account of memory effects carefully. We do not deal with such cases in the present paper.
}

If the off-diagonal elements of $\rho(\tau)$ can be further neglected, ${ }^{4}$ then (30) becomes

$$
\dot{\rho}_{m m}(\tau)=\sum_{k \neq m}\left[w_{m k}^{X}\left(\tau, \tau_{1}\right) \rho_{k k}(\tau)-w_{k m}^{X}\left(\tau, \tau_{1}\right) \rho_{m m}(\tau)\right]
$$

Here, we have introduced the transition rate matrix

$$
w_{m k}^{X}\left(\tau, \tau_{1}\right) \equiv \lambda^{2}\left|\mu_{m k}\right|^{2} \dot{\mathcal{F}}_{X}\left(E_{m}-E_{k} ; \tau, \tau_{1}\right),
$$

where

$$
\begin{aligned}
\dot{\mathcal{F}}_{X}\left(\Delta E ; \tau, \tau_{1}\right) \equiv & \int_{\tau_{1}}^{\tau} \mathrm{d} \tau^{\prime}\left[e^{-\mathrm{i} \Delta E\left(\tau^{\prime}-\tau\right)} G_{X}^{+}\left(x\left(\tau^{\prime}\right), x(\tau)\right)\right. \\
& \left.+e^{-\mathrm{i} \Delta E\left(\tau-\tau^{\prime}\right)} G_{X}^{+}\left(x(\tau), x\left(\tau^{\prime}\right)\right)\right]
\end{aligned}
$$

Equation (31) now has the standard form of the master equation.

If, in particular, $\dot{\mathcal{F}}^{\mathrm{eq}}(\Delta E) \equiv \lim _{\tau-\tau_{1} \rightarrow \infty} \dot{\mathcal{F}}_{X}\left(\Delta E ; \tau, \tau_{1}\right)$ satisfies the relation ${ }^{5}$

$$
\frac{\dot{\mathcal{F}}^{\mathrm{eq}}(\Delta E)}{\dot{\mathcal{F}}^{\mathrm{eq}}(-\Delta E)}=e^{-\beta \Delta E}
$$

the transition rate matrix satisfies the relation

$$
\frac{w_{m k}\left(\tau ; \tau_{1}\right)}{w_{k m}\left(\tau ; \tau_{1}\right)} \stackrel{\tau-\tau_{1} \rightarrow \infty}{\rightarrow} e^{-\beta\left(E_{m}-E_{k}\right)}
$$

Then, the distribution of the detector in equilibrium, $\rho^{\mathrm{eq}}$, may be determined by the detailed balance condition $\lim _{\tau-\tau_{1} \rightarrow \infty} w_{m k}\left(\tau ; \tau_{1}\right) \rho_{k k}^{\mathrm{eq}}=\lim _{\tau-\tau_{1} \rightarrow \infty} w_{k m}\left(\tau ; \tau_{1}\right) \rho_{m m}^{\mathrm{eq}}, \quad$ and we obtain

$$
\rho_{m m}^{\mathrm{eq}}=\frac{e^{-\beta E_{m}}}{Z} \quad\left(Z=\sum_{n} e^{-\beta E_{n}}\right)
$$

which is nothing but the Gibbs distribution at temperature $1 / \beta$.

We close this section by making a comment on the relationship between our formalism and the literature. One can easily show that the transition rate matrix $w_{m k}^{X}$, (32), is the $\tau$ derivative of

$$
\lambda^{2}\left|\mu_{m k}\right|^{2} \mathcal{F}_{X}\left(E_{m}-E_{k} ; \tau, \tau_{1}\right)
$$

\footnotetext{
${ }^{4}$ We will see in Sec. IV D that the off-diagonal elements of $\rho(\tau)$ can be set to zero without losing generality if the detector is a two-level system and $\mu$ has an off-diagonal form.

${ }^{5}$ The relation (34) indeed holds with $\beta=2 \pi \ell$ when $\rho^{\phi}\left(t_{1}\right)$ corresponds to the Bunch-Davies vacuum in de Sitter space. See Eq. (57).
} 
where

$\mathcal{F}_{X}\left(\Delta E ; \tau, \tau_{1}\right)=\int_{\tau_{1}}^{\tau} \mathrm{d} \tau^{\prime} \int_{\tau_{1}}^{\tau} \mathrm{d} \tau^{\prime \prime} e^{-\mathrm{i} \Delta E\left(\tau^{\prime}-\tau^{\prime \prime}\right)} G_{X}^{+}\left(x\left(\tau^{\prime}\right), x\left(\tau^{\prime \prime}\right)\right)$

In the literature (e.g., [3]), one often considers a process from an initial state $|k\rangle \otimes|\alpha\rangle$ at time $t_{1}$ (usually taken to be the infinite past) to a final state $|m\rangle \otimes|\beta\rangle$ at time $t$ and sums over the final states $|\beta\rangle$ of the scalar field. The transition probability has the same form as (37) if we set $X^{\phi}=\rho^{\phi}\left(t_{1}\right)=\rho_{I}^{\phi}\left(t_{1}\right)=|\alpha\rangle\langle\alpha|$ for which the Wightman function becomes $G_{X}^{+}\left(x, x^{\prime}\right)=\left\langle\alpha\left|\phi_{I}(x) \phi_{I}\left(x^{\prime}\right)\right| \alpha\right\rangle$. We thus again see that (32) represents the transition probability per unit proper time of the detector. $\mathcal{F}_{X}$ in (38) is often called the response function (see, e.g., [3]). As we have seen, $\mathcal{F}_{X}$ or its derivative $\dot{\mathcal{F}}_{X}$ does not depend on details of the detector and can be thoroughly determined by the Wightman function of the free scalar field.

\section{UNRUH-DEWITT DETECTOR IN DE SITTER SPACE}

In this section, we consider an Unruh-DeWitt detector in $d$-dimensional de Sitter space, which is weakly interacting with a massive scalar field $\phi(x)$ of mass $m$. We exclusively consider the Poincaré patch, denoting the time variable by $\eta$ :

$$
\mathrm{d} s^{2}=\ell^{2} \frac{-\mathrm{d} \eta^{2}+\mathrm{d} x^{2}}{\eta^{2}} \quad(-\infty<\eta<0)
$$

and set the classical trajectory of the detector to be the geodesic

$$
x^{\mu}(\tau)=\left(-\ell e^{-\tau / \ell}, \mathbf{0}\right)
$$

We denote by $\eta_{1}$ the time when the detector starts the interaction with the field $\phi(x)=\phi(\eta, x)$, which has the following form in the interaction picture [see (14)]:

$$
V_{I}(\eta)=\lambda \mu_{I}(\tau) \frac{\mathrm{d} \tau(\eta)}{\mathrm{d} \eta} \otimes \phi_{I}(x(\tau)) \theta\left(\eta-\eta_{1}\right)
$$

We will show that the density distribution of the detector exhibits a relaxation to the Gibbs distribution with $\beta=2 \pi \ell$ when the initial condition $X^{\phi}=\rho_{I}^{\phi}\left(\eta_{1}\right)$ satisfies the condition (23) and the corresponding Wightman function $G_{X}^{+}\left(x, x^{\prime}\right)$ has the same short distance behavior $\left(\left|\boldsymbol{x}-\boldsymbol{x}^{\prime}\right| \rightarrow 0\right.$ with $\left.\eta=\eta^{\prime}\right)$ as that of the Bunch-Davies vacuum, $G_{\mathrm{BD}}^{+}\left(x, x^{\prime}\right)$. In the following, we set the curvature radius $\ell=1$.

Let the initial density matrix $X^{\phi}=\rho_{I}^{\phi}\left(\eta_{1}\right)$ of the scalar field have the form

$$
X^{\phi}=|\mathrm{BD}\rangle\langle\mathrm{BD}|+\Delta X^{\phi},
$$

for which the Wightman function takes the form

$$
\begin{aligned}
G_{X}^{+}\left(x, x^{\prime}\right) & =\operatorname{Tr}_{\phi}\left(\phi_{I}(x) \phi_{I}\left(x^{\prime}\right) X^{\phi}\right) \\
& =G_{\mathrm{BD}}^{+}\left(x, x^{\prime}\right)+\Delta G^{+}\left(x, x^{\prime}\right) .
\end{aligned}
$$

We assume that the deformed Wightman function is invariant under spatial translations and rotations for fixed $\eta$ and $\eta^{\prime}$ and write its Fourier transform as $(k \equiv|\boldsymbol{k}|)$

$$
\begin{aligned}
G_{X}^{+}\left(\eta, \boldsymbol{x}, \eta^{\prime}, \boldsymbol{x}^{\prime}\right) \equiv & \int \frac{\mathrm{d}^{d-1} \boldsymbol{k}}{(2 \pi)^{d-1}} e^{\mathrm{i} \boldsymbol{k} \cdot\left(\boldsymbol{x}-\boldsymbol{x}^{\prime}\right)} G_{X, k}^{+}\left(\eta, \eta^{\prime}\right) \\
\equiv & \int \frac{\mathrm{d}^{d-1} \boldsymbol{k}}{(2 \pi)^{d-1}} e^{\mathrm{i} \boldsymbol{k} \cdot\left(\boldsymbol{x}-\boldsymbol{x}^{\prime}\right)} \\
& \times\left[G_{\mathrm{BD}, k}^{+}\left(\eta, \eta^{\prime}\right)+\Delta G_{k}^{+}\left(\eta, \eta^{\prime}\right)\right]
\end{aligned}
$$

This takes the following form for the geodesic (40) [with $\eta=-e^{-\tau}$ and $\eta^{\prime}=-e^{-\tau^{\prime}}$ ]:

$$
\begin{aligned}
G_{X}^{+}\left(x(\tau), x\left(\tau^{\prime}\right)\right)= & \int \frac{\mathrm{d}^{d-1} \boldsymbol{k}}{(2 \pi)^{d-1}} G_{X, k}^{+}\left(\eta, \eta^{\prime}\right) \\
= & \frac{2}{(4 \pi)^{\frac{d-1}{2}} \Gamma\left(\frac{d-1}{2}\right)} \int_{0}^{\infty} \mathrm{d} k k^{d-2} G_{X, k}^{+}\left(\eta, \eta^{\prime}\right) \\
\equiv & G_{\mathrm{BD}}^{+}\left(x(\tau), x\left(\tau^{\prime}\right)\right) \\
& +\Delta G^{+}\left(x(\tau), x\left(\tau^{\prime}\right)\right) .
\end{aligned}
$$

Note that both $G_{X, k}^{+}\left(\eta, \eta^{\prime}\right)$ and $G_{\mathrm{BD}, k}^{+}\left(\eta, \eta^{\prime}\right)$ [and thus $\Delta G_{k}^{+}\left(\eta, \eta^{\prime}\right)$ also] satisfy the homogeneous Klein-Gordon equation $\eta^{2} \ddot{f}(\eta)+(d-2) \eta \dot{f}(\eta)+\left(k^{2} \eta^{2}+m^{2}\right) f(\eta)=0$, with respect to each of the arguments $\eta$ and $\eta^{\prime}$. The solutions to this equation are given by linear combinations of $(-\eta)^{(d-1) / 2} H_{\nu}^{(a)}(-k \eta)(a=1,2)$, where $H_{\nu}^{(1,2)}(z)$ are the Hankel functions and

$$
\nu \equiv\left\{\begin{array}{l}
\sqrt{\left(\frac{d-1}{2}\right)^{2}-m^{2}} \quad\left(0<m<\frac{d-1}{2}\right), \\
i \sqrt{m^{2}-\left(\frac{d-1}{2}\right)^{2}} \quad\left(m \geq \frac{d-1}{2}\right) .
\end{array}\right.
$$

Thus the Wightman function is generically given by a linear combination of

$$
\left[(-\eta)\left(-\eta^{\prime}\right)\right]^{\frac{d-1}{2}} H_{\nu}^{(a)}(-k \eta) H_{\nu}^{(b)}\left(-k \eta^{\prime}\right) \quad(a, b=1,2) .
$$

For example, $G_{\mathrm{BD}, k}^{+}\left(\eta, \eta^{\prime}\right)$ is given by (see $\left.[3,7,12]\right)$

$$
G_{\mathrm{BD}, k}^{+}\left(\eta, \eta^{\prime}\right)=\frac{\pi}{4}\left[(-\eta)\left(-\eta^{\prime}\right)\right]^{\frac{d-1}{2}} H_{\nu}^{(1)}(-k \eta) H_{\nu}^{(2)}\left(-k \eta^{\prime}\right) .
$$

Because of the condition that $G_{X}^{+}\left(x, x^{\prime}\right)$ and $G_{\mathrm{BD}}^{+}\left(x, x^{\prime}\right)$ have the same short distance behavior, $\Delta G_{k}^{+}\left(\eta, \eta^{\prime}\right)$ takes the 


\section{MASTER EQUATION FOR THE UNRUH-DEWITT DETECTOR ...}

PHYSICAL REVIEW D 89, 064024 (2014)

following form ( $c_{0}$ is a constant with the dimension of time):

$$
\begin{aligned}
\Delta G_{k}^{+}\left(\eta, \eta^{\prime}\right)= & {\left[(-\eta)\left(-\eta^{\prime}\right)\right]^{\frac{d-1}{2}} } \\
& \times \sum_{a, b=1}^{2} f_{a b}\left(c_{0} k\right) H_{\nu}^{(a)}(-k \eta) H_{\nu}^{(b)}\left(-k \eta^{\prime}\right)
\end{aligned}
$$

with $^{6}$

$$
f_{a b}(z)=\text { const } z^{-\alpha_{a b}}\left[1+\mathcal{O}\left(z^{-1}\right)\right] \quad\left(\alpha_{a b}>0\right) .
$$

Note that we require only that the leading singularities be the same for $G_{X}^{+}\left(x, x^{\prime}\right)$ and $G_{\mathrm{BD}}^{+}\left(x, x^{\prime}\right)$ and the functions $f_{a b}(z)$ control the subleading singularities.

The derivative of the response function, $\dot{\mathcal{F}}_{X}$ [defined in Eq. (33)], can now be written as

$$
\dot{\mathcal{F}}_{X}\left(\Delta E ; \tau, \tau_{1}\right)=\dot{\mathcal{F}}_{\mathrm{BD}}\left(\Delta E ; \tau, \tau_{1}\right)+\Delta \dot{\mathcal{F}}\left(\Delta E ; \tau, \tau_{1}\right)
$$

where

$$
\begin{aligned}
\dot{\mathcal{F}}_{\mathrm{BD}}\left(\Delta E ; \tau, \tau_{1}\right) \equiv & \int_{-\left(\tau-\tau_{1}\right)}^{0} \mathrm{~d} s e^{-\mathrm{i} \Delta E s} G_{\mathrm{BD}}^{+}(x(\tau+s), x(\tau)) \\
& +\int_{0}^{\left(\tau-\tau_{1}\right)} \mathrm{d} s e^{-\mathrm{i} \Delta E s} G_{\mathrm{BD}}^{+}(x(\tau), x(\tau-s)),
\end{aligned}
$$

$$
\begin{aligned}
\Delta \dot{\mathcal{F}}\left(\Delta E ; \tau, \tau_{1}\right) \equiv & \int_{-\left(\tau-\tau_{1}\right)}^{0} \mathrm{~d} s e^{-\mathrm{i} \Delta E s} \Delta G^{+}(x(\tau+s), x(\tau)) \\
& +\int_{0}^{\left(\tau-\tau_{1}\right)} \mathrm{d} s e^{-\mathrm{i} \Delta E s} \Delta G^{+}(x(\tau), x(\tau-s)) .
\end{aligned}
$$

The integrals can be evaluated analytically as shown in Appendix E, and we find that they take the following asymptotic forms in the limit $\tau \rightarrow \infty$ [see Eqs. (E26) and (E19)]:

$$
\dot{\mathcal{F}}_{\mathrm{BD}}\left(\Delta E ; \tau, \tau_{1}\right) \sim \dot{\mathcal{F}}^{\mathrm{eq}}(\Delta E)+\text { const } e^{-\left(\frac{d-1}{2} \pm \nu \pm \mathrm{i} \Delta E\right)\left(\tau-\tau_{1}\right)},
$$

$$
\Delta \dot{\mathcal{F}}\left(\Delta E ; \tau, \tau_{1}\right) \sim \text { const } e^{-\alpha \tau}+\text { const } e^{-\left(\frac{d-1}{2} \pm \nu \pm \mathrm{i} \Delta E\right)\left(\tau-\tau_{1}\right)}
$$

with $\alpha \equiv \min _{a, b}\left(\alpha_{a b}\right)$.

The $\tau$-independent term $\dot{\mathcal{F}}^{\mathrm{eq}}(\Delta E)$ is given by [see Eq. (E21)]

$$
\dot{\mathcal{F}}^{\mathrm{eq}}(\Delta E)=\frac{e^{-\pi \ell \Delta E} \Gamma\left(\frac{\frac{d-1}{2}+\nu+\mathrm{i} \ell \Delta E}{2}\right) \Gamma\left(\frac{\frac{d-1}{2}-\nu+\mathrm{i} \ell \Delta E}{2}\right) \Gamma\left(\frac{\frac{d-1}{2}+\nu-\mathrm{i} \ell \Delta E}{2}\right) \Gamma\left(\frac{\frac{d-1}{2}-\nu-\mathrm{i} \ell \Delta E}{2}\right)}{8 \pi^{\frac{d+1}{2}} \Gamma\left(\frac{d-1}{2}\right)}
$$

which agrees with the known result obtained in Refs. [14,15] (we have restored the curvature radius $\ell$ ). One easily finds that this satisfies the relation

$$
\frac{\dot{\mathcal{F}}^{\mathrm{eq}}(\Delta E)}{\dot{\mathcal{F}}^{\mathrm{eq}}(-\Delta E)}=e^{-2 \pi \ell \Delta E}
$$

Thus, from the argument following (34), we confirm that, as $\tau$ becomes large, the density distribution $\rho_{m m}(\tau)$ approaches the Gibbs distribution at temperature $1 / 2 \pi \ell$ :

\footnotetext{
${ }^{6} \alpha_{a b}$ will need to be integers when imposing analyticity on the Wightman functions.
}

$$
\rho_{m m}^{\mathrm{eq}}=\frac{e^{-2 \pi \ell E_{m}}}{Z} \quad\left(Z=\sum_{n} e^{-2 \pi \ell E_{n}}\right)
$$

The $\tau$-dependent terms $e^{-\alpha \tau / \ell}$ and $e^{-\left[\left(\frac{d-1}{2} \pm \nu\right) / \ell \pm \mathrm{i} \Delta E\right] \tau}$ in the asymptotic forms (54) and (55) represent relaxation modes of the detector with relaxation times $\ell / \alpha$ and $|(d-1) / 2 \pm \operatorname{Re} \nu|^{-1} \ell$, respectively. Note that the modes with the latter relaxation time exist even when the initial state is the Bunch-Davies vacuum, in which we are not interested here. On the other hand, the mode with relaxation time $\ell / \alpha$ arises only when the initial state of the scalar field is deviated from the Bunch-Davies vacuum. We thus identify with $\ell / \alpha$ the relaxation time for the surrounding medium [see Fig. 1(b)] which relaxes from a nonequilibrium state to the equilibrium of temperature $1 / 2 \pi l$.

We close this section with writing down the full expression of (54) for comparison with the known results in the literature [see Eq. (E20)]: 


$$
\begin{aligned}
& \dot{\mathcal{F}}_{\mathrm{BD}}\left(\Delta E, \tau, \tau_{1}\right)=\dot{\mathcal{F}}^{\mathrm{eq}}(\Delta E)+\frac{e^{\mathrm{i} \pi \frac{d-1}{2}} e^{\mathrm{i} \pi \nu} e^{-\left(\frac{d-1}{2}+\nu-\mathrm{i} \Delta E\right)\left(\tau-\tau_{1}\right)}}{8 \pi^{\frac{d-1}{2}} \Gamma\left(\frac{d-1}{2}\right) \sin (\pi \nu)}{ }_{3} \hat{F}_{2}\left(\begin{array}{c}
\frac{d-1}{2}, \frac{\frac{d-1}{2}+\nu-\mathrm{i} \Delta E}{2}, \frac{d-1}{2}+\nu \\
1+\nu, \frac{\frac{d+3}{2}+\nu-\mathrm{i} \Delta E}{2}
\end{array} ; e^{-2\left(\tau-\tau_{1}\right)}\right) \\
& +\frac{e^{\mathrm{i} \pi \frac{d-1}{2}} e^{-\mathrm{i} \pi \nu} e^{-\left(\frac{d-1}{2}-\nu-\mathrm{i} \Delta E\right)\left(\tau-\tau_{1}\right)}}{8 \pi^{\frac{d-1}{2}} \Gamma\left(\frac{d-1}{2}\right) \sin (-\pi \nu)}{ }_{3} \hat{F}_{2}\left(\begin{array}{c}
\frac{d-1}{2}, \frac{\frac{d-1}{2}-\nu-\mathrm{i} \Delta E}{2}, \frac{d-1}{2}-\nu \\
1-\nu, \frac{\frac{d+3}{2}-\nu-\mathrm{i} \Delta E}{2}
\end{array} e^{-2\left(\tau-\tau_{1}\right)}\right) \\
& +\frac{e^{-\mathrm{i} \pi \frac{d-1}{2}} e^{-\mathrm{i} \pi \nu} e^{-\left(\frac{d-1}{2}+\nu+\mathrm{i} \Delta E\right)\left(\tau-\tau_{1}\right)}}{8 \pi^{\frac{d-1}{2}} \Gamma\left(\frac{d-1}{2}\right) \sin (\pi \nu)} \hat{F}_{2}\left(\begin{array}{c}
\frac{d-1}{2}, \frac{\frac{d-1}{2}+\nu+\mathrm{i} \Delta E}{2}, \frac{d-1}{2}+\nu \\
1+\nu, \frac{\frac{d+3}{2}+\nu+\mathrm{i} \Delta E}{2}
\end{array} e^{-2\left(\tau-\tau_{1}\right)}\right) \\
& \left.+\frac{e^{-\mathrm{i} \pi \frac{d-1}{2}} e^{\mathrm{i} \pi \nu} e^{-\left(\frac{d-1}{2}-\nu+\mathrm{i} \Delta E\right)\left(\tau-\tau_{1}\right)}}{8 \pi^{\frac{d-1}{2}} \Gamma\left(\frac{d-1}{2}\right) \sin (-\pi \nu)}{ }_{3} \hat{F}_{2}\left(\begin{array}{c}
\frac{d-1}{2}, \frac{\frac{d-1}{2}-\nu+\mathrm{i} \Delta E}{2}, \frac{d-1}{2}-\nu \\
1-\nu, \frac{\frac{d+3}{2}-\nu+\mathrm{i} \Delta E}{2}
\end{array} e^{-2\left(\tau-\tau_{1}\right)}\right)\right] .
\end{aligned}
$$

If we set $d=4$ and $\nu=1 / 2$, Eq. (59) correctly reproduces the result obtained in Sec. IV D of Ref. [14], where the case $\tau_{0}=-\infty$ is considered so that the higher-order corrections $\Delta \dot{\mathcal{F}}\left(\Delta E, \tau, \tau_{1}\right)$ are all zero.

\section{THE CASE OF THE INSTANTANEOUS GROUND STATE AT A FINITE PAST}

In this section, we consider the case where the initial state $X^{\phi}=\rho_{I}^{\phi}\left(\eta_{1}\right)$ is the instantaneous ground state at an earlier time $\eta_{0}$. In this case, $\Delta \dot{\mathcal{F}}\left(\Delta E ; \tau, \tau_{1}\right)$ can be calculated explicitly, and we will see that the relaxation time takes a universal value $\ell / 2$ (i.e., $\alpha=2$ ), irrespectively of the values of $\eta_{0}$ and $\eta_{1}$ or the form of interaction between the detector and the scalar.

\section{A. The instantaneous ground states}

Let $\left|0_{\eta_{0}}, \eta_{0}\right\rangle$ denote the Schrödinger picture state which is the instantaneous ground state at an early time $\eta_{0}$ (see Fig. 3). We then introduce the state $\left|0_{\eta_{0}}\right\rangle \equiv\left|0_{\eta_{0}}, \eta_{1}\right\rangle$ as an interaction picture state (or as a Heisenberg picture state of the free field theory) which is obtained by applying the free-field time evolution operator from $\eta_{0}$ to $\eta_{1}$ : $\left|0_{\eta_{0}}\right\rangle=\mathrm{T} \exp \left(-\mathrm{i} \int_{\eta_{0}}^{\eta_{1}} \mathrm{~d} \eta^{\prime} H^{\phi}\left(\eta^{\prime}\right)\right)\left|0_{\eta_{0}}, \eta_{0}\right\rangle$. In this section,

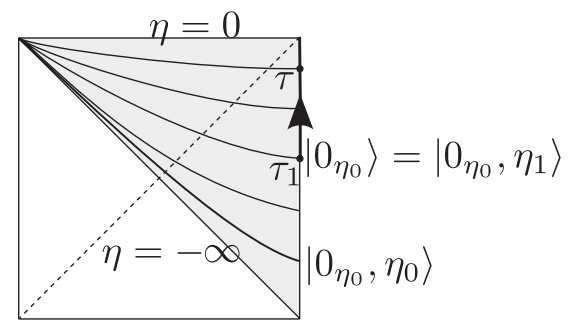

FIG. 3. The trajectory of an Unruh-DeWitt detector in the Penrose diagram of de Sitter space. The shaded region corresponds to the Poincaré patch, and the dashed line represents the future event horizon for the detector. The scalar field is in the ground state at time $\eta_{0}$. we set $X^{\phi}=\rho_{I}^{\phi}\left(\eta_{1}\right)=\left|0_{\eta_{0}}\right\rangle\left\langle 0_{\eta_{0}}\right|$. See [12] for a detailed discussion on defining such time-dependent ground states for a free scalar field in curved spacetimes.

Note that the one-point function vanishes: $\operatorname{Tr}_{\phi}\left(\phi_{I}(x) X^{\phi}\right)=\left\langle 0_{\eta_{0}}\left|\phi_{I}(x)\right| 0_{\eta_{0}}\right\rangle=0$. Then, with the approximations made in Sec. IIC, we have the master equation of the form [see (31)-(33)]

$\dot{\rho}_{m m}(\tau)=\sum_{k \neq m}\left[w_{m k}\left(\tau, \tau_{1} ; \eta_{0}\right) \rho_{k k}(\tau)-w_{k m}\left(\tau, \tau_{1} ; \eta_{0}\right) \rho_{m m}(\tau)\right]$

with the transition rate matrix

$$
w_{m k}\left(\tau, \tau_{1} ; \eta_{0}\right) \equiv \lambda^{2}\left|\mu_{m k}\right|^{2} \dot{\mathcal{F}}\left(E_{m}-E_{k} ; \tau, \tau_{1} ; \eta_{0}\right) .
$$

Here, the derivative of the response function is given by

$$
\begin{aligned}
\dot{\mathcal{F}}\left(\Delta E ; \tau, \tau_{1} ; \eta_{0}\right) \equiv & \int_{\tau_{1}}^{\tau} \mathrm{d} \tau^{\prime}\left[e^{-\mathrm{i} \Delta E\left(\tau^{\prime}-\tau\right)} G^{+}\left(x\left(\tau^{\prime}\right), x(\tau) ; \eta_{0}\right)\right. \\
& \left.+e^{-\mathrm{i} \Delta E\left(\tau-\tau^{\prime}\right)} G^{+}\left(x(\tau), x\left(\tau^{\prime}\right) ; \eta_{0}\right)\right]
\end{aligned}
$$

with the Wightman function

$$
G^{+}\left(x, x^{\prime} ; \eta_{0}\right) \equiv\left\langle 0_{\eta_{0}}\left|\phi_{I}(x) \phi_{I}\left(x^{\prime}\right)\right| 0_{\eta_{0}}\right\rangle .
$$

In the following, we compute $G^{+}\left(x, x^{\prime} ; \eta_{0}\right)$ for finite $\eta_{0}$ by using a technique developed in [12] and will find that $G^{+}\left(x, x^{\prime} ; \eta_{0}\right)$ indeed has the form (43) and (49) with

$$
\begin{aligned}
& f_{12}(z)=f_{21}(z)=\frac{\pi}{4}\left(\frac{d-2}{4}\right)^{2} z^{-2}+\mathcal{O}\left(z^{-4}\right), \\
& f_{11}(z)=f_{22}(z)=\mathcal{O}\left(z^{-4}\right) .
\end{aligned}
$$

Thus, $\alpha=2$ in this model, and the relaxation time of a medium surrounding the detector is given by $\ell / 2$. Since an explicit functional form can be obtained for $\Delta G^{+}\left(x, x^{\prime} ; \eta_{0}\right)$ 
in this model, we can further determine the coefficients of the damping term $e^{-\alpha \tau}$ in Eq. (55).

We comment that the instantaneous ground states $\left|0_{\eta_{0}}\right\rangle$ differ from the adiabatic vacuum state [16,17] (see also $[3,18])$ at second adiabatic order for finite $\eta_{0}$. This rapid departure may be expected from the fact that the condition of the validity of adiabatic approximation $\left[\omega_{k} \gg \dot{\omega}_{k} / \omega_{k}\right.$ with $\omega_{k}$ given by (73)] does not hold for some modes $\boldsymbol{k}$ if $\eta_{0}$ is finite. Actually, while the adiabatic vacuum state has the same asymptotic expansion as the Bunch-Davies vacuum, the wave function of an instantaneous ground state agrees with that of the Bunch-Davies vacuum only at the leading asymptotic order [see (C4)]. This observation then means that we cannot use the adiabatic subtraction scheme to regularize the expectation value of the energy-momentum tensor, $\left\langle T_{\mu \nu}\right\rangle$, for instantaneous ground states. In fact, it is shown in Refs. [19,20] that, in order for a state in $d$-dimensional de Sitter space to admit such adiabatic regularization of $\left\langle T_{\mu \nu}\right\rangle$, the state must be of $d$ th or higher adiabatic order; in other words, the wave function $\varphi_{k}(\eta)$ of the state must have the form

$$
\varphi_{k}(\eta)=(-\eta)^{\frac{d-1}{2}}\left[c_{1}(k) H_{\nu}^{(1)}(-k \eta)+c_{2}(k) H_{\nu}^{(2)}(-k \eta)\right]
$$

with $c_{1}(k)=$ const $+o\left(k^{-d}\right)$ and $c_{2}(k)=o\left(k^{-d}\right),{ }^{7}$ which is clearly different from the form given in (C4). We expect that one can yet use the point-splitting regularization to remove divergences of $\left\langle T_{\mu \nu}\right\rangle$ for instantaneous ground states, although one then needs to establish an algorithm to determine the finite part of $\left\langle T_{\mu \nu}\right\rangle$. This issue is beyond the scope of this paper and is left for future work.

\section{B. Wightman function for the instantaneous ground state}

In the Poincare patch, there is a translational invariance in the spatial directions, and we assume that all spatial directions are compactified with radius $L / 2 \pi{ }^{8}$ Then the wave vector $\boldsymbol{k}$ takes discrete values $\boldsymbol{k}=(2 \pi / L) \boldsymbol{n}$ with $\boldsymbol{n} \in \mathbb{Z}^{d-1}$. We denote $\boldsymbol{k}>0$ (or $\boldsymbol{k}<0$ ) if the first nonvanishing element of the vector $\boldsymbol{k}=\left(k_{1}, k_{2}, \ldots, k_{d-1}\right)$ is positive (or negative). Now, we consider the mode expansion

$$
\phi(x)=\phi(\eta, \boldsymbol{x})=\sum_{k \geq 0} \sum_{a} \phi_{k, a}(\eta) Y_{k, a}(\boldsymbol{x}),
$$

where the mode functions $\left\{Y_{\boldsymbol{k}, a}(\boldsymbol{x})\right\}$ are given by

\footnotetext{
${ }^{7}$ For such states, the exponents $\alpha_{a b}$ in (50) are greater than $d$, and thus the relaxation time will take a value less than $\ell / d$.

${ }^{8}$ The introduction of $L$ is for dealing with the zero mode carefully. $L$ will be taken to infinity in the end.
}

$$
\begin{aligned}
\boldsymbol{k}=0: Y_{\boldsymbol{k}=0, a=1} & \equiv \frac{1}{\sqrt{V}} \quad\left(V \equiv L^{d-1}\right), \\
\boldsymbol{k}>0: Y_{\boldsymbol{k}, a=1}(\boldsymbol{x}) & =\sqrt{\frac{2}{V}} \cos (\boldsymbol{k} \cdot \boldsymbol{x}), \\
Y_{\boldsymbol{k}, a=2}(\boldsymbol{x}) & =\sqrt{\frac{2}{V}} \sin (\boldsymbol{k} \cdot \boldsymbol{x}),
\end{aligned}
$$

which form a complete set of (real-valued) eigenfunctions of the spatial Laplacian $\Delta_{d-1}=\sum_{i=1}^{d-1} \partial_{i}^{2}$. Then, the Wightman function (27) is given by

$$
\begin{aligned}
G_{X}^{+}\left(x, x^{\prime}\right)= & \sum_{k \geq 0} G_{X, k}^{+}\left(\eta, \eta^{\prime}\right) \sum_{a} Y_{\boldsymbol{k}, a}(\boldsymbol{x}) Y_{\boldsymbol{k}, a}\left(\boldsymbol{x}^{\prime}\right) \\
= & \frac{1}{(2 \pi)^{\frac{d-1}{2}}\left|\boldsymbol{x}-\boldsymbol{x}^{\prime}\right|^{\frac{d-3}{2}}} \\
& \times \int_{0}^{\infty} \mathrm{d} k k^{\frac{d-1}{2}} J_{\frac{d-3}{2}}\left(k\left|\boldsymbol{x}-\boldsymbol{x}^{\prime}\right|\right) G_{X, k}^{+}\left(\eta, \eta^{\prime}\right)
\end{aligned}
$$

with

$$
G_{X, k}^{+}\left(\eta, \eta^{\prime}\right)=\left\langle 0_{\eta_{0}}\left|\phi_{k, a}(\eta) \phi_{k, a}\left(\eta^{\prime}\right)\right| 0_{\eta_{0}}\right\rangle
$$

where we have taken the limit $L \rightarrow \infty$ and integrated over angular variables in the second equality of Eq. (69).

With the mode expansion (66), the Hamiltonian (in the Heisenberg picture of the free field theory) is expressed as a sum of the Hamiltonians for independent harmonic oscillators:

$$
H(\eta)=\sum_{k, a} \omega_{k}(\eta) a_{\boldsymbol{k}, a}^{\dagger}(\eta) a_{\boldsymbol{k}, a}(\eta)+\mathrm{const}
$$

$$
a_{k, a}(\eta) \equiv \frac{(-\eta)^{-\frac{d-2}{2}}}{\sqrt{2}}\left[\omega_{k}^{1 / 2}(\eta) \phi_{k, a}(\eta)+\mathrm{i} \omega_{k}^{-1 / 2}(\eta) \dot{\phi}_{k, a}(\eta)\right]
$$

$$
\omega_{k}(\eta) \equiv \sqrt{m^{2}(-\eta)^{-2}+k^{2}}
$$

The instantaneous ground state $\left|0_{\eta_{0}}\right\rangle$ is then defined by the condition that $a_{k, a}\left(\eta_{0}\right)\left|0_{\eta_{0}}\right\rangle=0(\forall \boldsymbol{k}, a)$.

By expanding the operator $\phi_{k, a}(\eta)$ using the annihilation and creation operators $a_{\boldsymbol{k}, a}\left(\eta_{0}\right)$ and $a_{\boldsymbol{k}, a}^{\dagger}\left(\eta_{0}\right)$ as

$$
\phi_{\boldsymbol{k}, a}(\eta)=\varphi_{k}\left(\eta ; \eta_{0}\right) a_{\boldsymbol{k}, a}\left(\eta_{0}\right)+\varphi_{k}^{*}\left(\eta ; \eta_{0}\right) a_{\boldsymbol{k}, a}^{\dagger}\left(\eta_{0}\right),
$$

the ground state wave function $\varphi_{k}\left(\eta ; \eta_{0}\right)$ is given by (see [12]) 


$$
\begin{aligned}
\varphi_{k}\left(\eta ; \eta_{0}\right)= & -\frac{\pi}{2 \sqrt{2 \omega_{k}\left(\eta_{0}\right)}} \frac{(-\eta)^{\frac{d-1}{2}}}{\left(-\eta_{0}\right)^{-\frac{d-2}{2}}}\left[v_{0} J_{\nu}(-k \eta)\right. \\
& \left.-u_{0} N_{\nu}(-k \eta)\right]
\end{aligned}
$$

where $J_{\nu}(x)$ and $N_{\nu}(x)$ are the Bessel and Neumann functions, respectively, and $u_{0}$ and $v_{0}$ are given by

$$
\begin{aligned}
u_{0}= & -\left(-\eta_{0}\right)^{-\frac{d-1}{2}}\left[\left(\frac{d-1}{2}+\nu+\mathrm{i} \omega_{k}\left(\eta_{0}\right) \eta_{0}\right) J_{\nu}\left(-k \eta_{0}\right)\right. \\
& \left.+k \eta_{0} J_{1+\nu}\left(-k \eta_{0}\right)\right]
\end{aligned}
$$

$$
\begin{aligned}
v_{0}= & -\left(-\eta_{0}\right)^{-\frac{d-1}{2}}\left[\left(\frac{d-1}{2}+\nu+\mathrm{i} \omega_{k}\left(\eta_{0}\right) \eta_{0}\right) N_{\nu}\left(-k \eta_{0}\right)\right. \\
& \left.+k \eta_{0} N_{1+\nu}\left(-k \eta_{0}\right)\right] .
\end{aligned}
$$

If we expand the wave function $\varphi_{k}\left(\eta ; \eta_{0}\right)$ around $\eta_{0}=-\infty$, we find that the Wightman function for each mode,

$$
\begin{aligned}
G_{k}^{+}\left(\eta, \eta^{\prime} ; \eta_{0}\right) & =\left\langle 0_{\eta_{0}}\left|\phi_{k, a}(\eta) \phi_{k, a}\left(\eta^{\prime}\right)\right| 0_{\eta_{0}}\right\rangle \\
& =\varphi_{k}\left(\eta ; \eta_{0}\right) \varphi_{k}^{*}\left(\eta^{\prime} ; \eta_{0}\right),
\end{aligned}
$$

takes the following form (see Appendix C):

$$
\begin{aligned}
G_{k}^{+}\left(\eta, \eta^{\prime} ; \eta_{0}\right)= & \frac{\pi}{4}\left[(-\eta)\left(-\eta^{\prime}\right)\right]^{\frac{d-1}{2}}\left\{H_{\nu}^{(1)}(-k \eta) H_{\nu}^{(2)}\left(-k \eta^{\prime}\right)+\left(\frac{d-2}{4}\right)^{2} \frac{1}{\left(-k \eta_{0}\right)^{2}}\left[H_{\nu}^{(1)}(-k \eta) H_{\nu}^{(2)}\left(-k \eta^{\prime}\right)\right.\right. \\
& \left.+H_{\nu}^{(2)}(-k \eta) H_{\nu}^{(1)}\left(-k \eta^{\prime}\right)\right] \\
& +e^{2 \mathrm{i}\left[k \eta_{0}+\frac{\pi(1+2 \nu)}{4}\right]}\left[\mathrm{i} \frac{d-2}{4\left(-k \eta_{0}\right)}+\frac{\left(\nu^{2}-\frac{1}{4}\right)(d-3)-\left(\frac{d-2}{2}\right)^{2}-m^{2}}{4\left(-k \eta_{0}\right)^{2}}\right] H_{\nu}^{(1)}(-k \eta) H_{\nu}^{(1)}\left(-k \eta^{\prime}\right) \\
& +e^{-2 \mathrm{i}\left[k \eta_{0}+\frac{\pi(1+2 \nu)}{4}\right]}\left[-\mathrm{i} \frac{d-2}{4\left(-k \eta_{0}\right)}+\frac{\left(\nu^{2}-\frac{1}{4}\right)(d-3)-\left(\frac{d-2}{2}\right)^{2}-m^{2}}{4\left(-k \eta_{0}\right)^{2}}\right] H_{\nu}^{(2)}(-k \eta) H_{\nu}^{(2)}\left(-k \eta^{\prime}\right) \\
& \left.+\mathcal{O}\left(\left(-k \eta_{0}\right)^{-3}\right)\right\} .
\end{aligned}
$$

Here, the first term, which can be written as $\lim _{\eta_{0} \rightarrow-\infty} G_{k}^{+}\left(\eta, \eta^{\prime} ; \eta_{0}\right)$, is the Wightman function for the Bunch-Davies vacuum, $G_{\mathrm{BD}, k}^{+}\left(\eta, \eta^{\prime}\right)$. The last two terms include rapidly oscillating factors $e^{ \pm 2 i k \eta_{0}}$ and can be dropped from the expression, because they do not contribute to the integral in (69) for a sufficiently large $\left|\eta_{0}\right|$. We thus find that the Wightman function has the form

$$
G_{X, k}^{+}\left(\eta, \eta^{\prime} ; \eta_{0}\right)=G_{\mathrm{BD}, k}^{+}\left(\eta, \eta^{\prime}\right)+\Delta G_{k}^{+}\left(\eta, \eta^{\prime} ; \eta_{0}\right)
$$

with $^{9}$

$$
\begin{aligned}
& \Delta G_{k}^{+}\left(\eta, \eta^{\prime} ; \eta_{0}\right)= {\left[(-\eta)\left(-\eta^{\prime}\right)\right]^{\frac{d-1}{2}} f\left(-k \eta_{0}\right) } \\
& \times\left[H_{\nu}^{(1)}\left(-e^{\mathrm{i} \varepsilon} k \eta\right) H_{\nu}^{(2)}\left(-e^{-\mathrm{i} \varepsilon} k \eta^{\prime}\right)\right. \\
&\left.+H_{\nu}^{(2)}\left(-e^{-\mathrm{i} \varepsilon} k \eta\right) H_{\nu}^{(1)}\left(-e^{\mathrm{i} \varepsilon} k \eta^{\prime}\right)\right] \\
&+\mathcal{O}\left(\left(-k \eta_{0}\right)^{-4}\right), \\
& f(z) \equiv \frac{\pi}{4}\left(\frac{d-2}{4}\right)^{2} z^{-2} .
\end{aligned}
$$

\footnotetext{
${ }^{9}$ Terms of order $\left(-k \eta_{0}\right)^{-3}$ also disappear, since they necessarily include the rapidly oscillating factors.
}

Here, an infinitesimal positive constant $\varepsilon$ is introduced in order to make the $k$ integration in Eq. (69) finite (see footnote 11 in Appendix D). Then, comparing (81) and (82) with (50), we find that $\alpha=2$ and thus the relaxation time is $\ell / \alpha=\ell / 2$. Note that the reason why the relaxation time is given by $\ell / 2$ (not by $\ell$ ) is the disappearance of the order $\left(-k \eta_{0}\right)^{-1}$ terms from (79) due to highly oscillatory integrals.

We close this subsection with a comment that the state $\left|0_{\eta_{0}}\right\rangle$ does not have a de Sitter invariance, since it introduces an extra scale $\eta_{0}$ as a cutoff [even though the corresponding Wightman function has the same short distance behavior as $\left.G_{\mathrm{BD}}^{+}\left(x, x^{\prime}\right)\right]$. This can be seen from the fact that $G^{+}\left(x, x^{\prime} ; \eta_{0}\right)$ does not have a de Sitter-invariant form (see Appendix D).

\section{Higher-order corrections in $\dot{\mathcal{F}}$}

With the Wightman function (80), $\dot{\mathcal{F}}$ defined in (62) takes the form

$$
\dot{\mathcal{F}}\left(\Delta E, \tau, \tau_{1} ; \eta_{0}\right)=\dot{\mathcal{F}}_{\mathrm{BD}}\left(\Delta E ; \tau, \tau_{1}\right)+\Delta \dot{\mathcal{F}}\left(\Delta E ; \tau, \tau_{1} ; \eta_{0}\right),
$$

where $\dot{\mathcal{F}}_{\mathrm{BD}}\left(\Delta E ; \tau, \tau_{1}\right)$ and $\Delta \dot{\mathcal{F}}\left(\Delta E ; \tau, \tau_{1} ; \eta_{0}\right)$ are given by the integrals (52) and (53), respectively. The integrals 
can again be performed analytically as is done in Appendix E, and we obtain [see (54) and (55) and recall that $\alpha=2$ ]

$$
\dot{\mathcal{F}}_{\mathrm{BD}}\left(\Delta E, \tau, \tau_{1}\right) \sim \dot{\mathcal{F}}^{\mathrm{eq}}(\Delta E)+\text { const } e^{-\left(\frac{d-1}{2} \pm \nu \pm \mathrm{i} \Delta E\right)\left(\tau-\tau_{1}\right)},
$$

$$
\begin{aligned}
\Delta \dot{\mathcal{F}}\left(\Delta E, \tau, \tau_{1} ; \eta_{0}\right) \sim & e^{-2\left(\tau-\tau_{0}\right)} \Delta \dot{\mathcal{F}}^{(0)}(\Delta E) \\
& + \text { const } e^{-\left(\frac{d-1}{2} \pm \nu \pm \mathrm{i} \Delta E\right)\left(\tau-\tau_{1}\right)},
\end{aligned}
$$

where $\tau_{0} \equiv-\log \left(-\eta_{0}\right)$ and the coefficient of the leading term in $\Delta \dot{\mathcal{F}}\left(\Delta E, \tau, \tau_{1} ; \eta_{0}\right)$ is given by ${ }^{10}$

$$
\begin{aligned}
\Delta \dot{\mathcal{F}}^{(0)}(\Delta E)= & \left(\frac{d-2}{4}\right)^{2} \frac{\mathrm{i}}{\left.2^{5} \pi^{\frac{d-1}{2}} \Gamma \frac{d-1}{2}\right)}\left\{e ^ { \mathrm { i } \pi \frac { d - 2 } { 2 } } \left[\frac{e^{\mathrm{i} \pi \nu}}{\sin (\pi \nu)} \hat{F}_{2}\left(\begin{array}{c}
\frac{d-3}{2}, \frac{d-3}{2}+\nu \frac{\frac{d-1}{2}+\nu-2-\mathrm{i} \Delta E}{2} \\
1+\nu, \frac{\frac{d+3}{2}+\nu-2-\mathrm{i} \Delta E}{2}
\end{array} e^{-\mathrm{i} 0}\right)\right.\right. \\
& \left.+\frac{e^{-\mathrm{i} \pi \nu}}{\sin (-\pi \nu)} \hat{F}_{2}\left(\begin{array}{c}
\frac{d-3}{2}, \frac{d-3}{2}-\nu \frac{\frac{d-1}{2}-\nu-2-\mathrm{i} \Delta E}{2} \\
1-\nu, \frac{\frac{d+3}{2}-\nu-2-\mathrm{i} \Delta E}{2}
\end{array} e^{-\mathrm{i} 0}\right)\right]+e^{-\mathrm{i} \pi \frac{d-2}{2}}\left[\frac{e^{-\mathrm{i} \pi \nu}}{\sin (\pi \nu)}{ }_{3} \hat{F}_{2}\left(\begin{array}{c}
\frac{d-3}{2}, \frac{d-3}{2}+\nu, \frac{\frac{d-1}{2}+\nu-2-\mathrm{i} \Delta E}{2} \\
1+\nu, \frac{\frac{d+3}{2}+\nu-2-\mathrm{i} \Delta E}{2}
\end{array} ; e^{\mathrm{i} 0}\right)\right. \\
& \left.\left.+\frac{e^{\mathrm{i} \pi \nu}}{\sin (-\pi \nu)}{ }_{3} \hat{F}_{2}\left(\begin{array}{c}
\frac{d-3}{2}, \frac{d-3}{2}-\nu, \frac{\frac{d-1}{2}-\nu-2-\mathrm{i} \Delta E}{2} \\
1-\nu, \frac{\frac{d+3}{2} \nu-2-\mathrm{i} \Delta E}{2}
\end{array} e^{\mathrm{i} 0}\right)\right]\right\}+(\Delta E \rightarrow-\Delta E) .
\end{aligned}
$$

\section{Thermalization of a two-level detector in de Sitter space}

As we discussed in the preceding subsections, if we take the initial state for the scalar field to be the instantaneous ground state at a finite past, there exists a damping term in $\dot{\mathcal{F}}$ with the relaxation time $\ell / 2$. This behavior of $\dot{\mathcal{F}}$ is expected to represent the relaxation of the surrounding medium. In this subsection, as an analytically tractable example, we consider the case where the detector is a twolevel system with energy eigenvalues $E_{1}$ and $E_{2}$, with $\Delta E \equiv E_{2}-E_{1}>0$, and describe how the density distribution of the detector approaches the Gibbs distribution with relaxation time $\ell / 2$.

To proceed with the analysis, we set the following assumptions:

(i) The initial distribution of the detector, $\rho\left(\tau_{1}\right)$, is averaged over the initial proper time $\tau_{1}$ for the duration $\Delta \tau \gtrsim 1 / \Delta E$. This is for describing distributions at different energy levels to a good accuracy. As a consequence, the off-diagonal elements of $\rho\left(\tau_{1}\right)$ can be effectively set to zero, because they are oscillatory during that time.

(ii) The energy difference should be much larger than the natural energy scale of de Sitter space, $\Delta E \gg \ell^{-1}=1$, in order for relaxation modes with relaxation times of order $\ell$ to be observed.

(iii) The operator $\mu$ is off diagonal with respect to the basis $|m\rangle(m=1,2)$ :

$$
\mu=(\langle m|\mu| n\rangle)=\left(\begin{array}{cc}
0 & \mu_{12} \\
\mu_{12}^{*} & 0
\end{array}\right) .
$$

This is simply for making the following analysis much easier.
One can easily show that the master equation (30) decomposes into the diagonal and off-diagonal parts under assumption (iii). The off-diagonal part is given by linear differential equations of the form

$$
\left(\begin{array}{l}
\dot{\rho}_{12}(\tau) \\
\dot{\rho}_{21}(\tau)
\end{array}\right)=\mathcal{M}\left(\tau, \tau_{1}\right)\left(\begin{array}{c}
\rho_{12}(\tau) \\
\rho_{21}(\tau)
\end{array}\right)
$$

$\mathcal{M}\left(\tau, \tau_{1}\right)$

$$
\equiv\left(\begin{array}{cc}
\mathrm{i} \Delta E-\left|\mu_{12}\right|^{2} \dot{\mathcal{F}}\left(0 ; \tau, \tau_{1} ; \eta_{0}\right) & \mu_{12}^{2} \dot{\mathcal{F}}\left(0 ; \tau, \tau_{1} ; \eta_{0}\right) \\
\mu_{12}^{* 2} \dot{\mathcal{F}}\left(0 ; \tau, \tau_{1} ; \eta_{0}\right) & -\mathrm{i} \Delta E-\left|\mu_{12}\right|^{2} \dot{\mathcal{F}}\left(0 ; \tau, \tau_{1} ; \eta_{0}\right)
\end{array}\right),
$$

which can be integrated to

$$
\left(\begin{array}{c}
\rho_{12}(\tau) \\
\rho_{21}(\tau)
\end{array}\right)=\mathrm{T} \exp \left(\int_{\tau_{1}}^{\tau} \mathrm{d} \tau^{\prime} \mathcal{M}\left(\tau^{\prime}, \tau_{1}\right)\right)\left(\begin{array}{l}
\rho_{12}(\tau) \\
\rho_{21}(\tau)
\end{array}\right) .
$$

Since we can effectively set $\rho_{12}\left(\tau_{1}\right)=\rho_{21}\left(\tau_{1}\right)=0$ due to assumption (i), we can also set $\rho_{12}(\tau)=\rho_{21}(\tau)=0$ [assuming that $\mathcal{M}\left(\tau^{\prime}, \tau_{1}\right)$ changes slowly when averaging over $\left.\tau_{1}\right]$.

\footnotetext{
${ }_{3}^{10} \hat{F}_{2}$ is defined from the generalized hypergeometric function ${ }_{3} F_{2}$ (see Appendix B) as

${ }_{3} \hat{F}_{2}\left(\begin{array}{c}a_{1}, a_{2}, a_{3} \\ b_{1}, b_{2}\end{array} z\right) \equiv \frac{\Gamma\left(a_{1}\right) \Gamma\left(a_{2}\right) \Gamma\left(a_{3}\right)}{\Gamma\left(b_{1}\right) \Gamma\left(b_{2}\right)} \times{ }_{3} F_{2}\left(\begin{array}{c}a_{1}, a_{2}, a_{3} \\ b_{1}, b_{2}\end{array} z\right)$.
} 
The diagonal part can then be obtained from (31) and (32) as

$$
\left(\begin{array}{l}
\dot{\rho}_{11}(\tau) \\
\dot{\rho}_{22}(\tau)
\end{array}\right)=\left(\begin{array}{cc}
-w_{21}\left(\tau, \tau_{1} ; \eta_{0}\right) & w_{12}\left(\tau, \tau_{1} ; \eta_{0}\right) \\
w_{21}\left(\tau, \tau_{1} ; \eta_{0}\right) & -w_{12}\left(\tau, \tau_{1} ; \eta_{0}\right)
\end{array}\right)\left(\begin{array}{c}
\rho_{11}(\tau) \\
\rho_{22}(\tau)
\end{array}\right)
$$

with

$$
w_{m k}\left(\tau, \tau_{1} ; \eta_{0}\right)=\lambda^{2}\left|\mu_{m k}\right|^{2} \dot{\mathcal{F}}\left(E_{m}-E_{k} ; \tau, \tau_{1} ; \eta_{0}\right) .
$$

Using Eqs. (83)-(85) together with assumptions (i) and (ii), one can easily show that $\dot{\mathcal{F}}$ comes to take the following form after averaging over $\tau_{1}$ :

$$
\begin{aligned}
\dot{\mathcal{F}}_{\text {av }}\left(\Delta E ; \tau ; \eta_{0}\right) \equiv & \frac{1}{\Delta \tau} \int_{\tau_{1}}^{\tau_{1}+\Delta \tau} \mathrm{d} \tau_{1}^{\prime} \dot{\mathcal{F}}\left(\Delta E ; \tau, \tau_{1}^{\prime} ; \eta_{0}\right) \\
= & \dot{\mathcal{F}}^{\mathrm{eq}}(\Delta E)+e^{-2\left(\tau-\tau_{0}\right)} \Delta \dot{\mathcal{F}}^{(0)}(\Delta E) \\
& +\mathcal{O}\left(e^{-4\left(\tau-\tau_{0}\right)}\right),
\end{aligned}
$$

where $\dot{\mathcal{F}}^{\mathrm{eq}}(\Delta E)$ and $\Delta \dot{\mathcal{F}}^{(0)}(\Delta E)$ are given by (56) and (86), respectively. Note that the uninteresting $\tau_{1}$-dependent terms proportional to $e^{-\left(\frac{d-1}{2} \pm \nu \pm \mathrm{i} \Delta E\right)\left(\tau-\tau_{1}\right)}$ have totally disappeared from $\dot{\mathcal{F}}$ due to the averaging procedure [see (84) and (85)]. Replacing $\dot{\mathcal{F}}\left(\Delta E ; \tau, \tau_{1} ; \eta_{0}\right)$ in (92) by $\dot{\mathcal{F}}_{\text {av }}\left(\Delta E ; \tau ; \eta_{0}\right)$, we obtain the master equation for the diagonal elements of the form

$$
\left(\begin{array}{c}
\dot{\rho}_{11}(\tau) \\
\dot{\rho}_{22}(\tau)
\end{array}\right)=\left(\begin{array}{cc}
-w_{+}\left(\tau ; \tau_{0}\right) & w_{-}\left(\tau ; \tau_{0}\right) \\
w_{+}\left(\tau ; \tau_{0}\right) & -w_{-}\left(\tau ; \tau_{0}\right)
\end{array}\right)\left(\begin{array}{c}
\rho_{11}(\tau) \\
\rho_{22}(\tau)
\end{array}\right)
$$

with

$$
\begin{aligned}
w_{ \pm}\left(\tau ; \tau_{0}\right) \equiv & \lambda^{2}\left|\mu_{12}\right|^{2}\left[\dot{\mathcal{F}}^{\mathrm{eq}}( \pm \Delta E)+e^{-2\left(\tau-\tau_{0}\right)} \Delta \dot{\mathcal{F}}^{(0)}( \pm \Delta E)\right. \\
& \left.+\mathcal{O}\left(e^{-4\left(\tau-\tau_{0}\right)}\right)\right] .
\end{aligned}
$$

From a general argument following (56), we have already seen that, as $\tau$ becomes large, the density distribution $\rho_{m m}(\tau)$ approaches the Gibbs distribution at temperature $1 / 2 \pi \ell$ :

$$
\rho_{m m}^{\mathrm{eq}}=\frac{e^{-2 \pi \ell E_{m}}}{Z} \quad\left(Z=e^{-2 \pi \ell E_{1}}+e^{-2 \pi \ell E_{2}}\right) .
$$

In order to investigate how the detector relaxes to this equilibrium, we expand $\rho_{m m}(\tau)$ as $\rho_{m m}(\tau) \equiv \rho_{m m}^{\mathrm{eq}}+$ $\Delta \rho_{m m}(\tau)$ and keep small quantities to the first order, assuming that $\Delta \rho_{m m} / \rho_{m m}^{\mathrm{eq}} \ll 1$ and $e^{-2\left(\tau-\tau_{0}\right)} \ll 1$. Then (94) becomes

$$
\begin{aligned}
\frac{\mathrm{d}}{\mathrm{d} \tau} \Delta \rho_{11}(\tau)= & -\lambda^{2}\left|\mu_{12}\right|^{2}\left[e^{-2\left(\tau-\tau_{0}\right)} \Delta \dot{\mathcal{F}}^{(0)}(\Delta E) \tanh (\pi \Delta E)\right. \\
& \left.+\left(\dot{\mathcal{F}}^{\mathrm{eq}}(\Delta E)+\dot{\mathcal{F}}^{\mathrm{eq}}(-\Delta E)\right) \Delta \rho_{11}(\tau)\right]
\end{aligned}
$$

We used the fact that $\Delta \dot{\mathcal{F}}^{(0)}(\Delta E)$ is an even function of $\Delta E$ [see (86)] and $\Delta \rho_{11}=-\Delta \rho_{22}$. This equation can be solved easily, and we find that the relaxation behavior of the density distribution is given by

$$
\begin{aligned}
\Delta \rho_{11}(\tau)= & e^{-\lambda^{2}\left|\mu_{12}\right|^{2} A(\Delta E)\left(\tau-\tau_{2}\right)} \Delta \rho_{11}\left(\tau_{2}\right) \\
& -\frac{\lambda^{2}\left|\mu_{12}\right|^{2} \Delta \dot{\mathcal{F}}^{(0)}(\Delta E)}{\lambda^{2}\left|\mu_{12}\right|^{2} A(\Delta E)-2} \tanh (\pi \Delta E)\left(e^{-2\left(\tau-\tau_{2}\right)}\right. \\
& \left.-e^{-\lambda^{2}\left|\mu_{12}\right|^{2} A(\Delta E)\left(\tau-\tau_{2}\right)-2\left(\tau_{2}-\tau_{0}\right)}\right) .
\end{aligned}
$$

Here,

$$
A(\Delta E) \equiv \dot{\mathcal{F}}^{\mathrm{eq}}(\Delta E)+\dot{\mathcal{F}}^{\mathrm{eq}}(-\Delta E)
$$

and $\tau_{2}$ is an arbitrary proper time after $\tau_{1}\left(\tau_{2}>\tau_{1}\right)$, where the linear approximation is well justified.

We note that the function $A(\Delta E)$ has the following asymptotic form for large $|\Delta E|$ :

$$
A(\Delta E) \sim \frac{|\Delta E|^{d-3}}{2^{d-2} \pi^{\frac{d-3}{2}} \Gamma\left(\frac{d-1}{2}\right)} \quad(|\Delta E| \rightarrow \infty),
$$

as can be easily shown by using the asymptotic form of the Gamma function (Eq. 8.328-1 of [21]),

$$
\lim _{|y| \rightarrow \infty}|\Gamma(x+\mathrm{i} y)| \sim \sqrt{2 \pi} e^{-\frac{\pi}{2}|y|}|y|^{x-\frac{1}{2}} \quad(x, y \in \mathbb{R}) .
$$

We now consider a detector that satisfies the inequality

$$
\lambda^{2}\left|\mu_{12}\right|^{2}(\Delta E)^{d-3} \ell \gg 1 .
$$

Then, by using the asymptotic form (100), $A(\Delta E) \sim|\Delta E|^{d-3}$, and the inequality (102), the damping terms in (98) which are proportional to $e^{-\lambda^{2}\left|\mu_{12}\right|^{2} A(\Delta E)\left(\tau-\tau_{2}\right)}$ rapidly disappear from the expression, leaving only the term proportional to $e^{-2\left(\tau-\tau_{2}\right)}$ :

$$
\Delta \rho_{11}(\tau) \sim-\frac{\Delta \dot{\mathcal{F}}^{(0)}(\Delta E)}{A(\Delta E)} \tanh (\pi \ell \Delta E) e^{-2\left(\tau-\tau_{2}\right) / \ell},
$$

where we have restored the curvature radius $\ell$. Note that the coefficient $\Delta \dot{\mathcal{F}}^{(0)}(\Delta E) / A(\Delta E)$ does not depend on details of the detector (such as $\lambda \mu_{12}$ ). The remaining damping term in (103) corresponds to the desired relaxation mode with the relaxation time $\ell / 2$. Since only those rapidly disappearing terms depend on details of the detector, one may say that such a detector under consideration is an ideal detector, in the sense that it quickly loses its own 
nonequilibrium properties and gets adjusted to its environment almost instantaneously.

\section{CONCLUSION AND DISCUSSIONS}

In this paper, we have considered an Unruh-DeWitt detector staying in the Poincaré patch of de Sitter space. The main difference of our setup from those in the literature is that the scalar field (before interacting with the detector) is not in the Bunch-Davies vacuum (nor in the $\alpha$ vacuum). Then the Unruh-DeWitt detector behaves as if it is in a nonequilibrium environment. In order to deal with such situations, we first derived the master equation which describes a finite time evolution of the density matrix of an Unruh-DeWitt detector in arbitrary geometry.

We then applied the framework to de Sitter space. We showed that there exists a damping term in $\mathcal{F}$ with a relaxation time of the form $\ell / \alpha$ if the initial state of the scalar field is chosen such that the Wightman function takes the form (43) with (49) and (50). In particular, if we take the initial state to be the instantaneous ground state at a finite past, the relaxation time is always given by $\ell / 2$. We further gave an explicit description of the relaxation process for a two-level detector.

We here should stress again that we are not considering just the thermalization process of a detector dipped in a thermal bath. In fact, since the initial state is chosen to be different from the Bunch-Davies vacuum, the detector should initially behave as if it is in a medium which is not in thermodynamic equilibrium. As time goes on, the detector comes to behave as if it is in a thermal bath, since the difference of the initial state from the Bunch-Davies vacuum becomes irrelevant at later times (i.e., $\Delta \dot{\mathcal{F}} / \dot{\mathcal{F}}_{\mathrm{BD}} \ll 1$ at later times).

In reality, there can be many relaxation processes for such a detector, some of which are simply the processes where the detector gets adjusted to its environment. However, these processes usually depend on details of the detector and thus can be neglected by considering an ideal detector which quickly responds to changes in its environment. The terms proportional to $e^{-\lambda^{2}\left|\mu_{12}\right|^{2} A(\Delta E) \tau}$ in (98) actually represent such processes. On the other hand, there are terms including the factors $e^{-\left(\frac{d-1}{2} \pm \operatorname{Re} \nu\right) \tau}$ in $\dot{\mathcal{F}}$. This kind of term always exists even when the initial state is the Bunch-Davies vacuum and thus does not have relevance to the relaxation of the nonequilibrium medium depicted in Fig. 1(b). For the case of the two-level detector considered in Sec. IV D, such damping terms disappear from the expression after taking an average over the startup time $\tau_{1}$. There is also a damping term proportional to $e^{-\alpha \tau / \ell}$ [see (55)], which appears only when the initial state is different from the Bunch-Davies vacuum and thus describes the nonequilibrium dynamics of the surrounding medium. In particular, the relaxation time takes a universal value $\ell / 2$ (i.e., $\alpha=2$ ) for the instantaneous ground states at finite pasts. We expect that the relaxation time $\ell / 2$ gives a quantity representing the nonequilibrium thermodynamic character intrinsic to de Sitter space, just as the temperature of the final Gibbs distribution represents the Gibbons-Hawking temperature intrinsic to de Sitter space.

In the previous studies, thermal properties of de Sitter space have been investigated mainly in the context of equilibrium thermodynamics. The relaxation processes discussed in this paper may serve as examples related to the nonequilibrium dynamics of de Sitter space.

\section{ACKNOWLEDGMENTS}

We thank Y. Hamada, H. Ishimori, T. Kameyama, H. Kawai, K. Murase, K. Oda, and A. Ogasahara for useful discussions on the Unruh-DeWitt detector. This work was supported by the Grant-in-Aid for the Global COE program "The Next Generation of Physics, Spun from Universality and Emergence" from the Ministry of Education, Culture, Sports, Science and Technology (MEXT) of Japan. This work was also supported by MEXT (Grant No. 23540304).

\section{APPENDIX A: DERIVATION OF THE MASTER EQUATION}

In this Appendix, we derive the master equation (24). Since $\rho_{I}^{\text {tot }}(t)$ satisfies the time evolution equation (18), $\mathcal{P} \rho_{I}^{\text {tot }}(t)$ and $\mathcal{Q} \rho_{I}^{\text {tot }}(t)$ satisfy the differential equations (note that $\mathcal{P}+\mathcal{Q}=1$ )

$$
\frac{\mathrm{d}}{\mathrm{d} t} \mathcal{P} \rho_{I}^{\mathrm{tot}}(t)=-\mathrm{i} \mathcal{P} \operatorname{ad}_{V_{I}(t)} \mathcal{P} \rho_{I}^{\mathrm{tot}}(t)-\mathrm{i} \mathcal{P} \operatorname{ad}_{V_{I}(t)} \mathcal{Q} \rho_{I}^{\mathrm{tot}}(t),
$$

$$
\frac{\mathrm{d}}{\mathrm{d} t} \mathcal{Q} \rho_{I}^{\mathrm{tot}}(t)=-\mathrm{i} \mathcal{Q} \operatorname{ad}_{V_{I}(t)} \mathcal{P} \rho_{I}^{\mathrm{tot}}(t)-\mathrm{i} \mathcal{Q} \operatorname{ad}_{V_{I}(t)} \mathcal{Q} \rho_{I}^{\mathrm{tot}}(t) .
$$

The solution of (A2) is given by

$$
\begin{aligned}
\mathcal{Q} \rho_{I}^{\mathrm{tot}}(t)= & -i \int_{t_{1}}^{t} \mathrm{~d} t^{\prime} \mathrm{T} e^{-\mathrm{i} \int_{t^{\prime}}^{t} \mathrm{~d}^{\prime \prime} \mathcal{Q} \operatorname{ad}_{V_{I}\left(t^{\prime \prime}\right)}} \mathcal{Q} \operatorname{ad}_{V_{I}\left(t^{\prime}\right)} \mathcal{P} \rho_{I}^{\mathrm{tot}}\left(t^{\prime}\right) \\
& +\mathrm{T} e^{-\mathrm{i} \int_{t_{1}}^{t} \mathrm{~d}^{\prime} \mathcal{Q} \operatorname{ad}_{V_{I}\left(t^{\prime}\right)} \mathcal{Q} \rho_{I}^{\mathrm{tot}}\left(t_{1}\right),}
\end{aligned}
$$

and, substituting this to (A1) and using $(\mathrm{d} / \mathrm{d} t) \mathcal{P} \rho_{I}^{\text {tot }}(t)=$ $\left(\mathrm{d} \rho_{I}(t) / \mathrm{d} t\right) \otimes X^{\phi}$, we obtain 
MASAFUMI FUKUMA, SOTARO SUGISHITA, AND YUHO SAKATANI

$$
\begin{aligned}
\frac{\mathrm{d} \rho_{I}(t)}{\mathrm{d} t} \otimes X^{\phi}= & -\mathrm{i} \mathcal{P} \operatorname{ad}_{V_{I}(t)} \mathcal{P} \rho_{I}^{\mathrm{tot}}(t)-\mathrm{i} \mathcal{P} \operatorname{ad}_{V_{I}(t)} \\
& \times \mathrm{T} e^{-\mathrm{i} \int_{t_{1}}^{t} \mathrm{~d}^{\prime} \mathcal{Q} \operatorname{ad}_{V_{I}\left(t^{\prime}\right)} \mathcal{Q} \rho_{I}^{\mathrm{tot}}\left(t_{1}\right)} \\
& -\mathcal{P} \operatorname{ad}_{V_{I}(t)} \int_{t_{1}}^{t} \mathrm{~d} t^{\prime} \mathrm{T} e^{-\mathrm{i} \int_{t^{\prime}}^{t} \mathrm{~d}^{\prime \prime} \mathcal{Q} \operatorname{ad}_{V_{I}\left(t^{\prime \prime}\right)}} \\
& \times \mathcal{Q} \operatorname{ad}_{V_{I}\left(t^{\prime}\right)} \mathcal{P} \rho_{I}^{\mathrm{tot}}\left(t^{\prime}\right) .
\end{aligned}
$$

This equation can be simplified by noticing that $X^{\phi}$ can be chosen arbitrarily without changing the time evolution of $\rho_{I}(t)$. If we set $X^{\phi}=\rho^{\phi}\left(t_{1}\right)=\rho_{I}^{\phi}\left(t_{1}\right)$, we have Eq. (22): $\mathcal{P} \rho_{I}^{\text {tot }}\left(t_{1}\right)=\rho_{I}^{\text {tot }}\left(t_{1}\right), \mathcal{Q} \rho_{I}^{\text {tot }}\left(t_{1}\right)=0$. Thus, the second term on the right-hand side of (A4) vanishes. Furthermore, since the interaction has the factorized form $V_{I}(t)=\lambda \mathrm{d} \tau \mu_{I}(\tau) \otimes$ $\phi_{I}(x(\tau)) \theta\left(t-t_{1}\right)$ [Eq. (14)], we obtain

$\operatorname{Tr}_{\phi}\left(\operatorname{ad}_{V_{I}(t)} \mathcal{P} \rho_{I}^{\mathrm{tot}}(t)\right)=\lambda \frac{\mathrm{d} \tau}{\mathrm{d} t}\left[\mu_{I}(\tau), \rho_{I}(t)\right] \operatorname{Tr}_{\phi}\left(\phi_{I}(x(\tau)) \rho_{I}^{\phi}\left(t_{1}\right)\right)$,

which vanishes when the condition (23) holds. We thus find that the first term in (A4) also vanishes:

$$
\mathcal{P} \operatorname{ad}_{V_{I}(t)} \mathcal{P} \rho_{I}^{\text {tot }}(t)=\operatorname{Tr}_{\phi}\left(\operatorname{ad}_{V_{I}(t)} \mathcal{P} \rho_{I}^{\text {tot }}(t)\right) \otimes X^{\phi}=0
$$

The last equation (A6) shows that $\mathcal{Q} \operatorname{ad}_{V_{I}(t)} \mathcal{P} \rho_{I}^{\text {tot }}(t)=$ $\operatorname{ad}_{V_{I}(t)} \mathcal{P} \rho_{I}^{\text {tot }}(t)=\operatorname{ad}_{V_{I}(t)}\left(\rho_{I}(t) \otimes \rho_{I}^{\phi}\left(t_{1}\right)\right)$, and, thus, (A4) becomes the master equation (24).

\section{APPENDIX B: MEIJER'S $g$ FUNCTION AND GENERALIZED HYPERGEOMETRIC FUNCTION}

Meijer's $G$ function $G_{p, q}^{m, n}(0 \leq m \leq q, 0 \leq n \leq p)$ is defined by (see Sec. 9.302 of [21] for details on the choice of the contour $C$ )

$$
\begin{aligned}
& G_{p, q}^{m, n}\left(z \mid \begin{array}{l}
a_{1}, \ldots, a_{p} \\
b_{1}, \ldots, b_{q}
\end{array}\right) \\
& =\int_{C} \frac{\mathrm{d} s}{2 \pi i} \frac{\prod_{j=1}^{m} \Gamma\left(b_{j}-s\right) \prod_{j=1}^{n} \Gamma\left(1-a_{j}+s\right)}{\prod_{j=m+1}^{q} \Gamma\left(1-b_{j}+s\right) \prod_{j=n+1}^{p} \Gamma\left(a_{j}-s\right)} z^{s} .
\end{aligned}
$$

This function is invariant under the arbitrary permutation of a set $\left\{a_{1}, \ldots, a_{n}\right\},\left\{a_{n+1}, \ldots, a_{p}\right\},\left\{b_{1}, \ldots, b_{m}\right\}$, or $\left\{b_{m+1}, \ldots, b_{q}\right\}$. As a special case of the $G$ function, the generalized hypergeometric function is defined by (Eq. $9.34-8$ of [21])

$$
\begin{aligned}
{ }_{p} F_{q}\left(\begin{array}{l}
a_{1}, \ldots, a_{p} \\
b_{1}, \ldots, b_{q}
\end{array} ; z\right) \\
\quad \equiv \frac{\prod_{i=1}^{q} \Gamma\left(b_{i}\right)}{\prod_{i=1}^{p} \Gamma\left(a_{i}\right)} G_{p, q+1}^{1, p}\left(-z \mid \begin{array}{c}
1-a_{1}, \ldots, 1-a_{p} \\
0,1-b_{1}, \ldots, 1-b_{q}
\end{array}\right) \\
=\frac{\prod_{i=1}^{q} \Gamma\left(b_{i}\right)}{\prod_{i=1}^{p} \Gamma\left(a_{i}\right)} \int_{C} \frac{\mathrm{d} s}{2 \pi i} \frac{\Gamma(-s) \prod_{i=1}^{p} \Gamma\left(a_{i}+s\right)}{\prod_{i=1}^{q} \Gamma\left(b_{i}+s\right)}(-z)^{s} .
\end{aligned}
$$

For convenience, we define the following functions using the generalized hypergeometric functions:

$$
\hat{F}_{q}\left(\begin{array}{l}
a_{1}, \ldots, a_{p} \\
b_{1}, \ldots, b_{q}
\end{array} ;\right) \equiv \frac{\prod_{i=1}^{p} \Gamma\left(a_{i}\right)}{\prod_{i=1}^{q} \Gamma\left(b_{i}\right)} p F_{q}\left(\begin{array}{l}
a_{1}, \ldots, a_{p} \\
b_{1}, \ldots, b_{q}
\end{array} ;\right) .
$$

If no two $b_{i} \quad(1 \leq i \leq m)$ differ by an integer, Meijer's $G$ function $G_{p, q}^{m, n}$ with $p<q$, or $p=q$ and $m+n>p$, or $p=q$ and $m+n=p$ and $|z|<1$, can be expanded by using the generalized hypergeometric functions [22]:

$$
\begin{aligned}
& G_{p, q}^{m, n}\left(z \mid \begin{array}{l}
a_{1}, \ldots, a_{p} \\
b_{1}, \ldots, b_{q}
\end{array}\right) \\
& =\sum_{k=1}^{m} \frac{\prod_{i=1, i \neq k}^{m} \Gamma\left(b_{i}-b_{k}\right) \prod_{i=1}^{n} \Gamma\left(1-a_{i}+b_{k}\right)}{\prod_{i=n+1}^{p} \Gamma\left(a_{i}-b_{k}\right) \prod_{i=m+1}^{q} \Gamma\left(1-b_{i}+b_{k}\right)} z^{b_{k}} \\
& \times{ }_{p} F_{q-1}\left(\begin{array}{c}
1-a_{1}+b_{k}, \ldots, 1-a_{p}+b_{k} \\
1-b_{1}+b_{k}, \ldots, *, \ldots, 1-b_{q}+b_{k}
\end{array} ;(-1)^{p-m-n} z\right),
\end{aligned}
$$

where $*$ means that the $k$ th term has been omitted. In particular, $G_{3,3}^{2,2}$ is expanded as

$$
\begin{aligned}
& G_{3,3}^{2,2}\left(z \mid \begin{array}{l}
a_{1}, a_{2}, a_{3} \\
b_{1}, b_{2}, b_{3}
\end{array}\right) \\
& =z^{b_{1}} \frac{\Gamma\left(b_{2}-b_{1}\right) \Gamma\left(1-b_{2}+b_{1}\right)}{\Gamma\left(a_{3}-b_{1}\right) \Gamma\left(1-a_{3}+b_{1}\right)} \\
& \times_{3} \hat{F}_{2}\left(\begin{array}{c}
1-a_{1}+b_{1}, 1-a_{2}+b_{1}, 1-a_{3}+b_{1} \\
1-b_{2}+b_{1}, 1-b_{3}+b_{1}
\end{array} ;-z\right) \\
& +z^{b_{2}} \frac{\Gamma\left(b_{1}-b_{2}\right) \Gamma\left(1-b_{1}+b_{2}\right)}{\Gamma\left(a_{3}-b_{2}\right) \Gamma\left(1-a_{3}+b_{2}\right)} \\
& \times_{3} \hat{F}_{2}\left(\begin{array}{c}
1-a_{1}+b_{2}, 1-a_{2}+b_{2}, 1-a_{3}+b_{2} \\
1-b_{1}+b_{2}, 1-b_{3}+b_{2}
\end{array} ;-z\right) \text {. }
\end{aligned}
$$

Furthermore, one can show 
MASTER EQUATION FOR THE UNRUH-DEWITT DETECTOR ...

PHYSICAL REVIEW D 89, 064024 (2014)

$$
\begin{aligned}
G_{4,4}^{2,3}\left(z \mid \begin{array}{l}
a_{1}, a_{2}, a_{3}, a_{4} \\
b_{1}, b_{2}, b_{3}, a_{4}
\end{array}\right)= & z^{b_{1}} \frac{\Gamma\left(b_{2}-b_{1}\right) \Gamma\left(1-b_{2}+b_{1}\right)}{\Gamma\left(a_{4}-b_{1}\right) \Gamma\left(1-a_{4}+b_{1}\right)}{ }_{4} \hat{F}_{3}\left(\begin{array}{c}
1-a_{1}+b_{1}, 1-a_{2}+b_{1}, 1-a_{3}+b_{1}, 1-a_{4}+b_{1} ;-z \\
1-b_{2}+b_{1}, 1-b_{3}+b_{1}, 1-a_{4}+b_{1}
\end{array}\right) \\
& +z^{b_{2}} \frac{\Gamma\left(b_{1}-b_{2}\right) \Gamma\left(1-b_{1}+b_{2}\right)}{\Gamma\left(a_{4}-b_{2}\right) \Gamma\left(1-a_{4}+b_{2}\right)} \hat{F}_{3}\left(\begin{array}{c}
1-a_{1}+b_{2}, 1-a_{2}+b_{2}, 1-a_{3}+b_{2}, 1-a_{4}+b_{2} ;-z \\
1-b_{1}+b_{2}, 1-b_{3}+b_{2}, 1-a_{4}+b_{2}
\end{array}\right) \\
= & z^{b_{1}} \frac{\Gamma\left(b_{2}-b_{1}\right) \Gamma\left(1-b_{2}+b_{1}\right)}{\Gamma\left(a_{4}-b_{1}\right) \Gamma\left(1-a_{4}+b_{1}\right)}{ }_{3} \hat{F}_{2}\left(\begin{array}{c}
1-a_{1}+b_{1}, 1-a_{2}+b_{1}, 1-a_{3}+b_{1} ;-z \\
1-b_{2}+b_{1}, 1-b_{3}+b_{1}
\end{array}\right) \\
& +z^{b_{2}} \frac{\Gamma\left(b_{1}-b_{2}\right) \Gamma\left(1-b_{1}+b_{2}\right)}{\Gamma\left(a_{4}-b_{2}\right) \Gamma\left(1-a_{4}+b_{2}\right)} \hat{F}_{3}\left(\begin{array}{c}
1-a_{1}+b_{2}, 1-a_{2}+b_{2}, 1-a_{3}+b_{2} \\
1-b_{1}+b_{2}, 1-b_{3}+b_{2}
\end{array} ;\right)
\end{aligned}
$$

by using the following identity [which can be derived from (B2)]:

$$
{ }_{4} F_{3}\left(\begin{array}{c}
a_{1}, a_{2}, a_{3}, a_{4} \\
b_{1}, b_{2}, a_{4}
\end{array} ; z\right)={ }_{3} F_{2}\left(\begin{array}{c}
a_{1}, a_{2}, a_{3} \\
b_{1}, b_{2}
\end{array} z\right)
$$

From the above identities, one can show the following relations for $\sigma, \sigma^{\prime}= \pm 1$, and $\alpha \in \mathbb{R}$ :

$$
\begin{aligned}
& G_{3,3}^{2,2}\left(-e^{-\left(\sigma-\sigma^{\prime}\right) \mathrm{i} 0} x \mid \begin{array}{c}
-\frac{d-\alpha-3}{2}, \frac{\alpha-\frac{d-5}{2}+\nu \pm \mathrm{i} \Delta E}{2}, \nu-\frac{d-\alpha-3}{2} \\
\nu, 0, \frac{\alpha-\frac{d-1}{2}+\nu \pm \mathrm{i} \Delta E}{2}
\end{array}\right) \\
& =-\frac{\left(-e^{-\left(\sigma-\sigma^{\prime}\right) \mathrm{i} 0} x\right)^{\nu} \sin \left(\pi \frac{d-1-\alpha}{2}\right)}{\sin (\pi \nu)}{ }_{3} \hat{F}_{2}\left(\begin{array}{c}
\frac{d-1-\alpha}{2}, \frac{d-1-\alpha}{2}+\nu, \frac{\frac{d-1}{2}+\nu-\alpha \mp \mathrm{i} \Delta E}{2} \\
1+\nu, \frac{\frac{d+3}{2}+\nu-\alpha \mp \mathrm{i} \Delta E}{2}
\end{array} ; e^{-\left(\sigma-\sigma^{\prime}\right) \mathrm{i} 0} x\right) \\
& -\frac{\sin \left(\pi \frac{d-1-\alpha-2 \nu}{2}\right)}{\sin (-\pi \nu)}{ }_{3} \hat{F}_{2}\left(\begin{array}{c}
\frac{d-1-\alpha}{2}, \frac{d-1-\alpha}{2}-\nu, \frac{\frac{d-1}{2}-\nu-\alpha \mp \mathrm{i} \Delta E}{2} \\
1-\nu, \frac{\frac{d+3}{2}-\nu-\alpha \mp \mathrm{i} \Delta E}{2}
\end{array} ; e^{-\left(\sigma-\sigma^{\prime}\right) \mathrm{i} 0} x\right), \\
& G_{4,4}^{2,3}\left(-e^{-\left(\sigma-\sigma^{\prime}\right) \mathrm{i} 0} x \mid \begin{array}{c}
-\frac{d-\alpha-3}{2}, \frac{\alpha-\frac{d-5}{2}+\nu \pm \mathrm{i} \Delta E}{2}, \nu-\frac{d-\alpha-3}{2}, \nu-\frac{d-\alpha-4}{2} \\
\nu, 0, \frac{\alpha-\frac{d-1}{2}+\nu \pm \mathrm{i} \Delta E}{2}, \nu-\frac{d-\alpha-4}{2}
\end{array}\right) \\
& =\frac{\left(-e^{-\left(\sigma-\sigma^{\prime}\right) \mathrm{i} 0} x\right)^{\nu} \cos \left(\pi \frac{d-1-\alpha}{2}\right)}{\sin (\pi \nu)}{ }_{3} \hat{F}_{2}\left(\begin{array}{c}
\frac{d-1-\alpha}{2}, \frac{d-1-\alpha}{2}+\nu, \frac{\frac{d-1}{2}+\nu-\alpha \mp \mathrm{i} \Delta E}{2} \\
1+\nu, \frac{\frac{d+3}{2}+\nu-\alpha \mp \mathrm{i} \Delta E}{2}
\end{array} ; e^{-\left(\sigma-\sigma^{\prime}\right) \mathrm{i} 0} x\right) \\
& +\frac{\cos \left(\pi \frac{d-1-\alpha-2 \nu}{2}\right)}{\sin (-\pi \nu)}{ }_{3} \hat{F}_{2}\left(\begin{array}{c}
\frac{d-1-\alpha}{2}, \frac{d-1-\alpha}{2}-\nu, \frac{\frac{d-1}{2}-\nu-\alpha \mp \mathrm{i} \Delta E}{2} \\
1-\nu, \frac{\frac{d+3}{2}-\nu-\alpha \mp \mathrm{i} \Delta E}{2}
\end{array} ; e^{-\left(\sigma-\sigma^{\prime}\right) \mathrm{i} 0} x\right)
\end{aligned}
$$

from which we obtain the following formula for $x>0$ :

$$
\begin{aligned}
G_{3,3}^{2,2}\left(-e^{-\mathrm{i}\left(\sigma-\sigma^{\prime}\right) 0} x \mid \begin{array}{c}
-\frac{d-\alpha-3}{2}, \frac{\alpha-\frac{d-5}{2} \pm \mathrm{i} \Delta E+\nu}{2}, \nu-\frac{d-\alpha-3}{2} \\
\nu, 0, \frac{\alpha-\frac{d-5}{2} \pm \mathrm{i} \Delta E+\nu}{2}-1
\end{array}\right)+\mathrm{i} \sigma G_{4,4}^{2,3}\left(-e^{-\mathrm{i}\left(\sigma-\sigma^{\prime}\right) 0} x \mid \begin{array}{c}
-\frac{d-\alpha-3}{2}, \frac{\alpha-\frac{d-5}{2}+\nu \pm \mathrm{i} \Delta E}{2}, \nu-\frac{d-\alpha-3}{2}, \nu-\frac{d-\alpha-4}{2} \\
\nu, 0, \frac{\alpha-\frac{d-5}{2}+\nu \pm \mathrm{i} \Delta E}{2}-1, \nu-\frac{d-\alpha-4}{2}
\end{array}\right) \\
=e^{\sigma i \pi \frac{d-\alpha}{2}}\left[\frac{\left(-e^{-\left(\sigma-\sigma^{\prime}\right) \mathrm{i} 0}\right)^{\nu} x^{\nu}}{\sin (\pi \nu)}{ }_{3} \hat{F}_{2}\left(\begin{array}{c}
\frac{d-1-\alpha}{2}, \frac{d-1-\alpha}{2}+\nu, \frac{\frac{d-1}{2}+\nu-\alpha \mp \mathrm{i} \Delta E}{2} \\
1+\nu, \frac{\frac{d+3}{2}+\nu-\alpha \mp \mathrm{i} \Delta E}{2}
\end{array} e^{-\mathrm{i}\left(\sigma-\sigma^{\prime}\right) 0} x\right)\right. \\
\left.\quad+\frac{e^{-\sigma i \pi \nu}}{\sin (-\pi \nu)}{ }_{3} \hat{F}_{2}\left(\begin{array}{c}
\frac{d-1-\alpha}{2}, \frac{d-1-\alpha}{2}-\nu, \frac{\frac{d-1}{2}-\nu-\alpha \mp \mathrm{i} \Delta E}{2} \\
1-\nu, \frac{\frac{d+3}{2}-\nu-\alpha \mp \mathrm{i} \Delta E}{2}
\end{array} e^{-\mathrm{i}\left(\sigma-\sigma^{\prime}\right) 0} x\right)\right] .
\end{aligned}
$$




\section{MASAFUMI FUKUMA, SOTARO SUGISHITA, AND YUHO SAKATANI}

Another useful formula can be obtained by using the identities [23]

$$
\begin{aligned}
& { }_{3} \hat{F}_{2}\left(\begin{array}{c}
a_{1}, a_{2}, a_{3} \\
b_{1}, b_{2}
\end{array} ; z\right)=(-z)^{-a_{1}} \frac{\Gamma\left(a_{1}\right) \Gamma\left(a_{2}-a_{1}\right) \Gamma\left(a_{3}-a_{1}\right)}{\Gamma\left(b_{1}-a_{1}\right) \Gamma\left(b_{2}-a_{1}\right)}{ }_{3} F_{2}\left(\begin{array}{c}
a_{1}, a_{1}-b_{1}+1, a_{1}-b_{2}+1 \\
a_{1}-a_{2}+1, a_{1}-a_{3}+1
\end{array} ; z^{-1}\right) \\
& +(-z)^{-a_{2}} \frac{\Gamma\left(a_{2}\right) \Gamma\left(a_{1}-a_{2}\right) \Gamma\left(a_{3}-a_{2}\right)}{\Gamma\left(b_{1}-a_{2}\right) \Gamma\left(b_{2}-a_{2}\right)}{ }_{3} F_{2}\left(\begin{array}{c}
a_{2}, a_{2}-b_{1}+1, a_{2}-b_{2}+1 \\
a_{2}-a_{1}+1, a_{2}-a_{3}+1
\end{array} ; z^{-1}\right) \\
& +(-z)^{-a_{3}} \frac{\Gamma\left(a_{3}\right) \Gamma\left(a_{1}-a_{3}\right) \Gamma\left(a_{2}-a_{3}\right)}{\Gamma\left(b_{1}-a_{3}\right) \Gamma\left(b_{2}-a_{3}\right)}{ }_{3} F_{2}\left(\begin{array}{c}
a_{3}, a_{3}-b_{1}+1, a_{3}-b_{2}+1 \\
a_{3}-a_{1}+1, a_{3}-a_{2}+1
\end{array} ; z^{-1}\right) \\
& {\left[a_{1}-a_{2} \notin \mathbb{Z}, a_{1}-a_{3} \notin \mathbb{Z}, a_{2}-a_{3} \notin \mathbb{Z}, \quad \text { and } z \notin(0,1)\right] \text {, }} \\
& { }_{3} F_{2}\left(\begin{array}{c}
a_{1}, a_{2}, 0 \\
b_{1}, b_{2}
\end{array} ; z\right)=1
\end{aligned}
$$

from which one can show

$$
\begin{aligned}
& { }_{3} \hat{F}_{2}\left(\begin{array}{c}
\frac{d-1}{2}, \frac{d-1}{2} \pm \nu, \frac{\frac{d-1}{2} \pm \nu+\mathrm{i} \Delta E}{2} \\
1 \pm \nu, \frac{\frac{d+3}{2} \pm \nu+\mathrm{i} \Delta E}{2}
\end{array} ; z\right)=(-z)^{-\frac{1}{2}\left(\frac{d-1}{2} \pm \nu+\mathrm{i} \Delta E\right)} \sin \left(\pi \frac{\frac{d-1}{2} \mp \nu+\mathrm{i} \Delta E}{2}\right) \frac{\Gamma\left(\frac{\frac{d-1}{2}+\nu+\mathrm{i} \Delta E}{2}\right) \Gamma\left(\frac{\frac{d-1}{2}-\nu+\mathrm{i} \Delta E}{2}\right) \Gamma\left(\frac{\frac{d-1}{2}+\nu-\mathrm{i} \Delta E}{2}\right) \Gamma\left(\frac{\frac{d-1}{2}-\nu-\mathrm{i} \Delta E}{2}\right)}{\pi} \\
& +(-z)^{-\frac{d-1}{2}} \frac{\sin \left(\pi \frac{d-1 \mp 2 \nu}{2}\right)}{\sin (\mp \pi \nu)}{ }_{3} \hat{F}_{2}\left(\begin{array}{c}
\frac{d-1}{2}, \frac{d-1}{2} \mp \nu, \frac{\frac{d-1}{2} \mp \nu-\mathrm{i} \Delta E}{2} \\
1 \mp \nu, \frac{\frac{d+3}{2} \mp \nu-\mathrm{i} \Delta E}{2}
\end{array} ; z^{-1}\right) \\
& +(-z)^{-\frac{d-1 \pm 2 \nu}{2}} \frac{\sin \left(\pi \frac{d-1}{2}\right)}{\sin ( \pm \pi \nu)}{ }_{3} \hat{F}_{2}\left(\begin{array}{c}
\frac{d-1}{2}, \frac{d-1}{2} \pm \nu, \frac{\frac{d-1}{2} \pm \nu-\mathrm{i} \Delta E}{2} \\
1 \pm \nu, \frac{\frac{d+3}{2} \pm \nu-\mathrm{i} \Delta E}{2}
\end{array} ; z^{-1}\right)
\end{aligned}
$$

Using this equality, the following formula can be shown to hold:

$$
\begin{aligned}
& -\frac{e^{-\mathrm{i} \pi\left(\frac{d-1}{2}+\nu\right)}}{\sin (\pi \nu)}{ }_{3} \hat{F}_{2}\left(\begin{array}{c}
\frac{d-1}{2}, \frac{d-1}{2}+\nu, \frac{\frac{d-1}{2}+\nu+\mathrm{i} \Delta E}{2} \\
1+\nu, \frac{\frac{d+3}{2}+\nu+\mathrm{i} \Delta E}{2}
\end{array} ; e^{i 0}\right)-\frac{e^{-\mathrm{i} \pi\left(\frac{d-1}{2}-\nu\right)}}{\sin (-\pi \nu)}{ }_{3} \hat{F}_{2}\left(\begin{array}{c}
\frac{d-1}{2}, \frac{d-1}{2}-\nu, \frac{\frac{d-1}{2}-\nu+\mathrm{i} \Delta E}{2} \\
1-\nu, \frac{\frac{d+3}{2}-\nu+\mathrm{i} \Delta E}{2}
\end{array} ; e^{\mathrm{i} 0}\right) \\
& =e^{-\pi \Delta E} \frac{\Gamma\left(\frac{\frac{d-1}{2}+\nu+\mathrm{i} \Delta E}{2}\right) \Gamma\left(\frac{\frac{d-1}{2}-\nu+\mathrm{i} \Delta E}{2}\right) \Gamma\left(\frac{\frac{d-1}{2}+\nu-\mathrm{i} \Delta E}{2}\right) \Gamma\left(\frac{\frac{d-1}{2}-\nu-\mathrm{i} \Delta E}{2}\right)}{\pi}+\frac{e^{\mathrm{i} \pi\left(\frac{d-1}{2}+\nu\right)}}{\sin (\pi \nu)} 3 \hat{F}_{2}\left(\begin{array}{c}
\frac{d-1}{2}, \frac{d-1}{2}+\nu, \frac{\frac{d-1}{2}+\nu-\mathrm{i} \Delta E}{2} \\
1+\nu, \frac{\frac{d+3}{2}+\nu-\mathrm{i} \Delta E}{2}
\end{array} ; e^{-\mathrm{i} 0}\right) \\
& +\frac{e^{\mathrm{i} \pi\left(\frac{d-1}{2}-\nu\right)}}{\sin (-\pi \nu)}{ }_{3} \hat{F}_{2}\left(\begin{array}{c}
\frac{d-1}{2}, \frac{d-1}{2}-\nu, \frac{\frac{d-1}{2}-\nu-\mathrm{i} \Delta E}{2} \\
1-\nu, \frac{\frac{d+3}{2}-\nu-\mathrm{i} \Delta E}{2}
\end{array} ; e^{-\mathrm{i} 0}\right)
\end{aligned}
$$

\section{APPENDIX C: DERIVATION OF EQ. (79)}

We derive the asymptotic form (79) of the Wightman function for each mode:

$$
G_{k}^{+}\left(\eta, \eta^{\prime} ; \eta_{0}\right)=\varphi_{k}\left(\eta ; \eta_{0}\right) \varphi_{k}^{*}\left(\eta^{\prime} ; \eta_{0}\right)
$$

By introducing $z \equiv-k \eta_{0}$ and $\theta(z) \equiv z-(\pi / 4)(1+2 \nu)$, and using the asymptotic form of the Bessel and Neumann functions around $z=\infty$ (i.e., $\eta_{0}=-\infty$ ) (see Sec. 8.451 of [21]), $u_{0}$ has the expansion

$$
\begin{aligned}
u_{0}= & \mathrm{i} \sqrt{\frac{2 k}{\pi}}\left(-\eta_{0}\right)^{-\frac{d-1}{2}}\left\{e^{-\mathrm{i} \theta(z)}\left[1+i \frac{\frac{d-2}{2}-\left(\nu^{2}-\frac{1}{4}\right)}{2 z}-\frac{\left(\nu^{2}-\frac{1}{4}\right)\left(\nu^{2}-\frac{1}{4}-(d-2)-2 m^{2}\right)}{8 z^{2}}\right]\right. \\
& \left.+e^{\mathrm{i} \theta(z)}\left[-i \frac{d-2}{4 z}+\frac{\left(\nu^{2}-\frac{1}{4}\right)(d-4)-2 m^{2}}{8 z^{2}}\right]+\mathcal{O}\left(z^{-3}\right)\right\} .
\end{aligned}
$$




\section{MASTER EQUATION FOR THE UNRUH-DEWITT DETECTOR ...}

$v_{0}$ can be obtained from this expression by replacing $\theta(z)$ with $\theta(z)-\pi / 2$ :

$$
\begin{aligned}
v_{0}= & -\sqrt{\frac{2 k}{\pi}}\left(-\eta_{0}\right)^{-\frac{d-1}{2}}\left\{e ^ { - i \theta ( z ) } \left[1+\mathrm{i} \frac{\frac{d-2}{2}-\left(\nu^{2}-\frac{1}{4}\right)}{2 z}\right.\right. \\
& \left.-\frac{\left(\nu^{2}-\frac{1}{4}\right)\left(\nu^{2}-\frac{1}{4}-(d-2)-2 m^{2}\right)}{8 z^{2}}\right] \\
& -e^{\mathrm{i} \theta(z)}\left[-\mathrm{i} \frac{d-2}{4 z}+\frac{\left(\nu^{2}-\frac{1}{4}\right)(d-4)-2 m^{2}}{8 z^{2}}\right] \\
& \left.+\mathcal{O}\left(z^{-3}\right)\right\}
\end{aligned}
$$

With the $u_{0}$ and $v_{0}$ given above, the wave function (75) is written as

$$
\begin{aligned}
\varphi_{k}\left(\eta ; \eta_{0}\right)= & \frac{\sqrt{\pi}}{2}(-\eta)^{\frac{d-1}{2}}\left\{e ^ { - \mathrm { i } \theta ( z ) } \left[1+\mathrm{i} \frac{\frac{d-2}{2}-\left(\nu^{2}-\frac{1}{4}\right)}{2 z}\right.\right. \\
& \left.-\frac{\left.\left(\nu^{2}-\frac{1}{4}\right)\left(\nu^{2}-\frac{1}{4}-(d-2)\right)\right]}{8 z^{2}}\right] H_{\nu}^{(1)}(-k \eta) \\
& -e^{\mathrm{i} \theta(z)}\left[-\mathrm{i} \frac{d-2}{4 z}+\frac{\left(\nu^{2}-\frac{1}{4}\right)(d-4)-2 m^{2}}{8 z^{2}}\right] \\
& \left.\times H_{\nu}^{(2)}(-k \eta)+\mathcal{O}\left(z^{-3}\right)\right\}
\end{aligned}
$$

Then, by substituting this to (C1), we obtain Eq. (79).

\section{APPENDIX D: WIGHTMAN FUNCTION}

\section{Analytic expression for the Wightman function}

The Wightman function for the instantaneous ground state,

$$
\begin{aligned}
G^{+}\left(x, x^{\prime} ; \eta_{0}\right)= & \frac{1}{(2 \pi)^{\frac{d-1}{2}}\left|\boldsymbol{x}-\boldsymbol{x}^{\prime}\right|^{\frac{d-3}{2}}} \\
& \times \int_{0}^{\infty} d k k^{\frac{d-1}{2}} J_{\frac{d-3}{2}}\left(k\left|\boldsymbol{x}-\boldsymbol{x}^{\prime}\right|\right) G_{k}^{+}\left(\eta, \eta^{\prime} ; \eta_{0}\right),
\end{aligned}
$$

has the expansion of the form

$$
G^{+}\left(x, x^{\prime} ; \eta_{0}\right)=\sum_{n=0}^{\infty} \sum_{a, b=1}^{2} c_{a, b}^{(n)}\left(\eta_{0}\right) G_{a, b}^{(n)}\left(x, x^{\prime}\right),
$$

where

$$
\begin{aligned}
G_{a, b}^{(n)}\left(x, x^{\prime}\right) \equiv & \frac{\left[(-\eta)\left(-\eta^{\prime}\right)\right]^{\frac{d-1}{2}}}{(2 \pi)^{\frac{d-1}{2}}\left|\boldsymbol{x}-\boldsymbol{x}^{\prime}\right|^{\frac{d-3}{2}}} \int_{0}^{\infty} \mathrm{d} k k^{\frac{d-1}{2}-n} J_{\frac{d-3}{2}}\left(k\left|\boldsymbol{x}-\boldsymbol{x}^{\prime}\right|\right) \\
& \times H_{\nu}^{(a)}\left(-e^{i \sigma_{a} \varepsilon} k \eta\right) H_{\nu}^{(b)}\left(-e^{i \sigma_{b} \varepsilon} k \eta^{\prime}\right),
\end{aligned}
$$

$c_{1,2}^{(0)}=\pi / 4, \quad c_{2,1}^{(0)}=c_{1,1}^{(0)}=c_{2,2}^{(0)}=0, \quad c_{a, b}^{(1)}=0$,

$c_{1,2}^{(2)}=c_{2,1}^{(2)}=\frac{\pi}{4}\left(\frac{d-2}{4}\right)^{2}\left(-\eta_{0}\right)^{-2}, \quad c_{1,1}^{(2)}=c_{2,2}^{(2)}=0$

with

$$
\sigma_{a} \equiv\left\{\begin{array}{cc}
+1 & (a=1) \\
-1 & (a=2)
\end{array}\right.
$$

Here, an infinitesimal positive constant $\varepsilon$ is introduced in order to make the $k$ integration finite. ${ }^{11}$ Note that the $n=1$ terms will totally disappear as a consequence of ignoring the highly oscillating terms.

In the following, we derive an analytic expression for the integral (D3). We take care of only terms with $a \neq b$, since terms with $a=b$ do not contribute to the Wightman function at least for $n \leq 2$ [see (D4)].

By using the identity $K_{\nu}\left(e^{-\mathrm{i} \sigma_{a} \frac{\pi}{2}} z\right)=$ $\left(\mathrm{i} \sigma_{a} \pi / 2\right) e^{\sigma_{a} \frac{\mathrm{i} \pi \nu}{2}} H_{\nu}^{(a)}(z)$, we first obtain the relation

$$
\begin{aligned}
& \int_{0}^{\infty} d k k^{\frac{d-3}{2}+1-n} J_{\frac{d-3}{2}}\left(k\left|\boldsymbol{x}-\boldsymbol{x}^{\prime}\right|\right) H_{\nu}^{(a)}(-k \eta) H_{\nu}^{(b)}\left(-k \eta^{\prime}\right) \\
& =-\frac{4 \sigma_{a} \sigma_{b}}{\pi^{2}} e^{-\left(\sigma_{a}+\sigma_{b}\right) \frac{\mathrm{i} \pi \nu}{2}} \int_{0}^{\infty} d k k^{\frac{d-3}{2}+1-n} J_{\frac{d-3}{2}}\left(k\left|\boldsymbol{x}-\boldsymbol{x}^{\prime}\right|\right) \\
& \quad \times K_{\nu}\left(-e^{-\mathrm{i} \sigma_{a} \frac{\pi}{2}} k \eta\right) K_{\nu}\left(-e^{-\mathrm{i} \sigma_{b} \frac{\pi}{2}} k \eta^{\prime}\right)
\end{aligned}
$$

Then, using the formula [29]

$$
\begin{aligned}
& \int_{0}^{\infty} \mathrm{d} k k^{\mu+1-n} K_{\nu}(a k) K_{\nu}(b k) J_{\mu}(c k) \\
& =\frac{2^{\mu-1-n}}{\Gamma(\mu+1)} \frac{c^{\mu}}{b^{2 \xi}}\left[(a / b)^{\nu} \Gamma(\xi) \Gamma(\xi+\nu) \Gamma(-\nu)\right. \\
& \quad \times F_{4}\left(\xi, \xi+\nu ; 1+\mu, 1+\nu ;-c^{2} / b^{2} ; a^{2} / b^{2}\right) \\
& \quad+(a / b)^{-\nu} \Gamma(\xi) \Gamma(\xi-\nu) \Gamma(\nu) \\
& \left.\quad \times F_{4}\left(\xi, \xi-\nu ; 1+\mu, 1-\nu ;-c^{2} / b^{2} ; a^{2} / b^{2}\right)\right] \\
& \quad[\xi \equiv \mu+1-(n / 2), \nu \notin \mathbb{Z}],
\end{aligned}
$$

we obtain the following expression for $G_{a, b}^{(n)}\left(x, x^{\prime}\right)$ :

\footnotetext{
${ }^{11}$ It is known that a delicate treatment is required in regularizing the integral to obtain the response function $\mathcal{F}$ [24-28]. However, we expect that the current regularization is sufficient to obtain its derivative $\dot{\mathcal{F}}$, since it gives a de Sitter-invariant form in the limit $\eta_{0} \rightarrow-\infty$ and reproduces all the known results in the literature as special cases. See comments following (86) and (59).
} 


$$
\begin{aligned}
G_{a, b}^{(n)}\left(x, x^{\prime}\right)= & \frac{\left(-\sigma_{a} \sigma_{b}\right) e^{-\left(\sigma_{a}+\sigma_{b}\right) \frac{\mathrm{i} \pi \nu}{2}}}{2^{n} \pi^{\frac{d+3}{2}} e^{-\mathrm{i} \pi \sigma_{b} \frac{d-1-n}{2}} \Gamma\left(\frac{d-1}{2}\right)}\left(\frac{-\eta}{-\eta^{\prime}}\right)^{\frac{d-1}{2}}\left(-\eta^{\prime}\right)^{n} \\
& \times\left[\left(\frac{-\eta}{-\eta^{\prime}}\right)^{\nu} e^{-\left(\sigma_{a}-\sigma_{b}\right) \frac{\mathrm{i} u}{2}} \Gamma\left(\frac{d-1-n}{2}+\nu\right)\right. \\
& \times \Gamma\left(\frac{d-1-n}{2}\right) \Gamma(-\nu) \\
& \times F_{4}\left(\frac{d-1-n}{2}+\nu, \frac{d-1-n}{2} ; \frac{d-1}{2}, 1+\nu ;\right. \\
& \left.\left.\frac{e^{-\mathrm{i} \sigma_{b} 0}\left|\boldsymbol{x}-\boldsymbol{x}^{\prime}\right|^{2}}{\left(-\eta^{\prime}\right)^{2}} ; \frac{e^{-\mathrm{i} \sigma_{a} \pi}(-\eta)^{2}}{e^{-\mathrm{i} \sigma_{b} \pi}\left(-\eta^{\prime}\right)^{2}}\right)+(\nu \rightarrow-\nu)\right] .
\end{aligned}
$$

Here, $F_{4}(a, b ; c, d ; x, y)$ is Appell's hypergeometric function given by

$$
\begin{gathered}
F_{4}(a, b ; c, d ; x, y) \equiv \sum_{m=0}^{\infty} \sum_{n=0}^{\infty} \frac{(a)_{m+n}(b)_{m+n}}{m ! n !(c)_{m}(d)_{n}} x^{m} y^{n} \\
{\left[|x|^{1 / 2}+|y|^{1 / 2}<1\right],}
\end{gathered}
$$

$$
(x)_{n} \equiv \Gamma(x+n) / \Gamma(x) \text {. }
$$

For $Z\left(x, x^{\prime}\right) \equiv\left[\eta^{2}+\eta^{\prime 2}-\left|\boldsymbol{x}-\boldsymbol{x}^{\prime}\right|^{2}\right] /\left(2 \eta \eta^{\prime}\right)<1$, the series in (D9) does not converge, and the analytic continuation should be performed (see Eq. 9.185 of [21] for the integral representation of the Mellin-Barnes type).

In the limit $\left|\boldsymbol{x}-\boldsymbol{x}^{\prime}\right| \rightarrow 0$, one can show, by using the identity

$$
F_{4}(a, b ; c, d ; 0, y) \equiv \sum_{n=0}^{\infty} \frac{(a)_{n}(b)_{n}}{n !(d)_{n}} y^{n}={ }_{2} F_{1}\left(\begin{array}{c}
a, b \\
d
\end{array} ; y\right)
$$

$$
\begin{aligned}
\lim _{\left|x-x^{\prime}\right| \rightarrow 0} G_{a, b}^{(n)}\left(x, x^{\prime}\right)= & \frac{\left(\sigma_{a} \sigma_{b}\right) e^{-\left(\sigma_{a}+\sigma_{b}\right) \frac{\mathrm{i} \nu}{2}}}{2^{n} \pi^{\frac{d+1}{2}} e^{-\mathrm{i} \pi \sigma_{b} \frac{d-1-n}{2}} \Gamma\left(\frac{d-1}{2}\right)}\left(\frac{-\eta}{-\eta^{\prime}}\right)^{\frac{d-1}{2}}\left(-\eta^{\prime}\right)^{n}\left[\left(\frac{-\eta}{-\eta^{\prime}}\right)^{\nu} \frac{e^{-\left(\sigma_{a}-\sigma_{b}\right) \frac{\mathrm{i} \nu}{2}}}{\sin (\pi \nu)}{ }_{2} \hat{F}_{1}\left(\begin{array}{c}
\frac{d-1-n}{2}+\nu, \frac{d-1-n}{2} \\
1+\nu
\end{array} \frac{e^{-\mathrm{i} \sigma_{a}(\pi-0)}(-\eta)^{2}}{e^{-\mathrm{i} \sigma_{b}(\pi-0)}\left(-\eta^{\prime}\right)^{2}}\right)\right. \\
& \left.+\left(\frac{-\eta}{-\eta^{\prime}}\right)^{-\nu} \frac{e^{\left(\sigma_{a}-\sigma_{b}\right) \frac{\mathrm{in} \nu}{2}}}{\sin (-\pi \nu)} \hat{F}_{1}\left(\begin{array}{c}
\frac{d-1-n}{2}-\nu, \frac{d-1-n}{2} \\
1-\nu
\end{array} ; \frac{e^{-\mathrm{i} \sigma_{a}(\pi-0)}(-\eta)^{2}}{e^{-\mathrm{i} \sigma_{b}(\pi-0)}\left(-\eta^{\prime}\right)^{2}}\right)\right] \\
= & \frac{4}{\pi} \frac{\left(-\sigma_{a} \sigma_{b}\right) e^{\frac{\mathrm{i} \pi}{2}\left(\sigma_{a}+\sigma_{b}\right)\left(\frac{d-1-n}{2}-\nu\right)} \Gamma\left(\frac{d-1-n}{2}\right)}{(4 \pi)^{\frac{d}{2}} \Gamma\left(\frac{d-1}{2}\right)}\left[(-\eta)\left(-\eta^{\prime}\right)\right]^{\frac{n}{2}} \hat{F}_{2}\left(\begin{array}{c}
\frac{d-1-n}{2}+\nu, \frac{d-1-n}{2}-\nu \\
\frac{d-n}{2}
\end{array}\right)
\end{aligned}
$$

with

$$
u_{a, b} \equiv \frac{e^{-\mathrm{i} \sigma_{a}(\pi-0)}(-\eta)^{2}+e^{-\mathrm{i} \sigma_{b}(\pi-0)}\left(-\eta^{\prime}\right)^{2}}{2 e^{-\mathrm{i}\left(\sigma_{a}+\sigma_{b}\right)(\pi-0)}(-\eta)\left(-\eta^{\prime}\right)} .
$$

Here, in the last equality, we have used formulas 9.132-2 and 9.134-3 of Ref. [21].

We comment that (D12) can be obtained more directly by performing an integration (D3) with setting $\left|\boldsymbol{x}-\boldsymbol{x}^{\prime}\right|=0$ in advance:

$$
\begin{aligned}
\lim _{\left|x-x^{\prime}\right| \rightarrow 0} G_{a, b}^{(n)}\left(x, x^{\prime}\right)= & \frac{2\left[(-\eta)\left(-\eta^{\prime}\right)\right]^{\frac{d-1}{2}}}{(4 \pi)^{\frac{d-1}{2}} \Gamma\left(\frac{d-1}{2}\right)} \int_{0}^{\infty} \mathrm{d} k k^{d-2-n} H_{\nu}^{(a)}\left(-e^{\mathrm{i} \sigma_{a} \varepsilon} k \eta\right) H_{\nu}^{(b)}\left(-e^{\mathrm{i} \sigma_{b} \varepsilon} k \eta^{\prime}\right), \\
= & \frac{\left(-\sigma_{a} \sigma_{b}\right) e^{-\left(\sigma_{a}+\sigma_{b}\right) \frac{\mathrm{i} \mu \nu}{2}} \Gamma\left(\frac{d-1-n}{2}\right) \Gamma\left(\frac{d-1-n}{2}-\nu\right)}{2^{n} \pi^{\frac{d-3}{2}} \Gamma\left(\frac{d-1}{2}\right)}(-\eta)^{n} e^{\mathrm{i} \pi \sigma_{a} \frac{d-1-n}{2}} e^{\left(\sigma_{a}-\sigma_{b}\right) \frac{\mathrm{i} \pi \nu}{2}} \\
& \times\left(\frac{-\eta}{-\eta^{\prime}}\right)^{-\frac{d-1}{2} \nu}{ }_{2} \hat{F}_{1}\left(\begin{array}{c}
\frac{d-1-n}{2}+\nu, \frac{d-1-n}{2} \\
1+\nu
\end{array} 1-\frac{e^{-\mathrm{i} \sigma_{b}(\pi-0)}\left(-\eta^{\prime}\right)^{2}}{e^{-\mathrm{i} \sigma_{a}(\pi-0)}(-\eta)^{2}}\right),
\end{aligned}
$$

where we have used formula 6.576-4 of [21]. It can be found that the last expression actually coincides with the first line on the right-hand side of (D12) by using formula $9.132-1$ of [21].
Note that the $n$ th-order Wightman function $G_{a, b}^{(n)}\left(x, x^{\prime}\right)$ is not de Sitter invariant for $n>0$, because it has an extra factor $\left[(-\eta)\left(-\eta^{\prime}\right)\right]^{n / 2}$ which multiplies a function of the de Sitter-invariant variable $u_{a, b}$. 

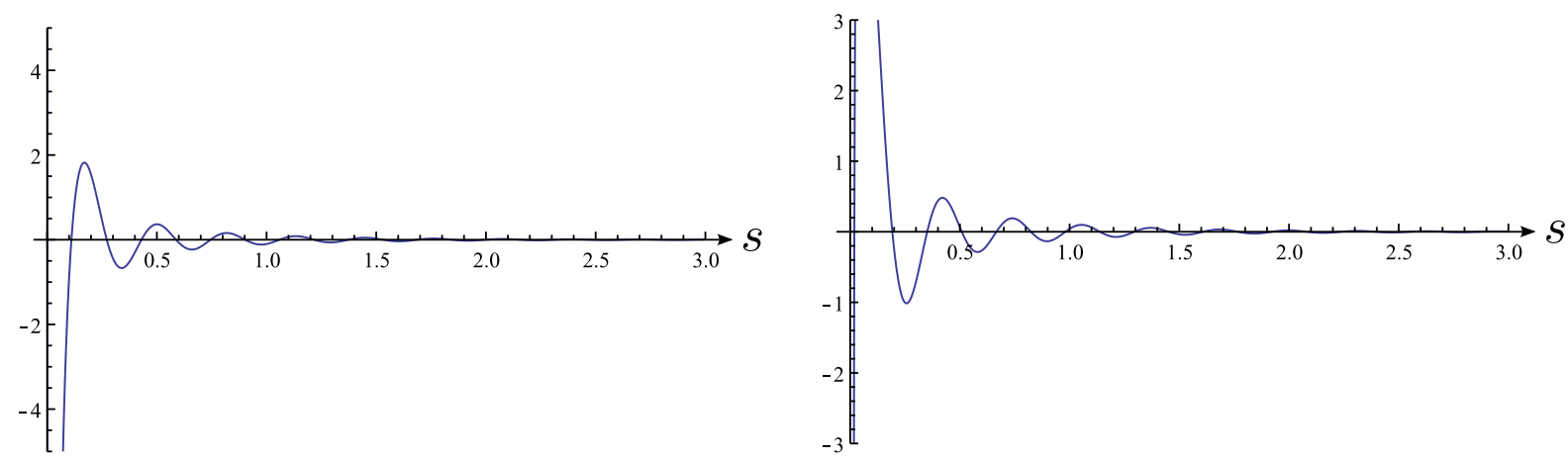

FIG. 4 (color online). The real part (left) and the imaginary part (right) of the Wightman function $G^{+}\left(x(\tau), x(\tau-s) ; \eta_{0}\right)$ for $d=4$, $\nu=20 \mathrm{i}$ (heavy mass case), and $\tau-\tau_{0}=50$.
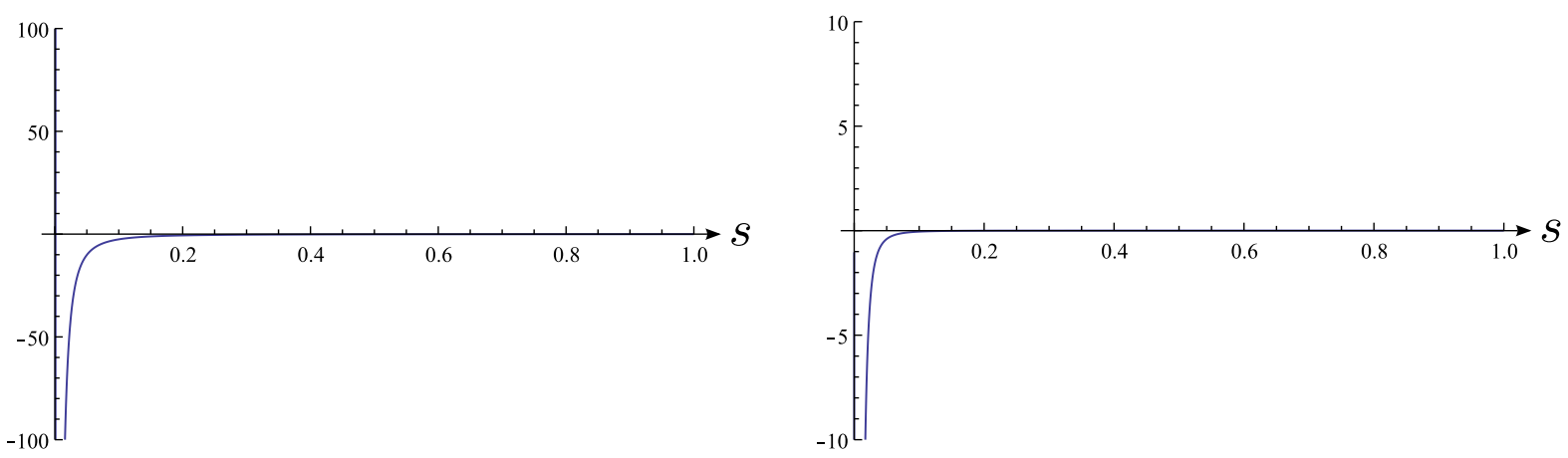

FIG. 5 (color online). The real part (left) and the imaginary part (right) of the Wightman function $G^{+}\left(x(\tau), x(\tau-s) ; \eta_{0}\right)$ for $d=4$, $\nu=0$, and $\tau-\tau_{0}=50$.

\section{Behavior of the Wightman function}

In order to understand the behavior of the Wightman function $^{12}$

$$
\begin{aligned}
& G^{+}(\left.x(\tau), x(\tau-s) ; \eta_{0}\right) \\
& \simeq G_{1,2}^{(0)}(x(\tau), x(\tau-s))+\frac{\pi}{4}\left(\frac{d-2}{4}\right)^{2}\left(-\eta_{0}\right)^{-2} \\
& \quad \times\left[G_{1,2}^{(2)}(x(\tau), x(\tau-s))+G_{2,1}^{(2)}(x(\tau), x(\tau-s))\right],
\end{aligned}
$$

\footnotetext{
${ }^{12}$ The Wightman function has the following asymptotic form for large $s$ :

$$
\begin{aligned}
G^{+}\left(x(\tau), x(\tau-s) ; \eta_{0}\right) \sim & \frac{e^{-\mathrm{i} \pi\left(\frac{d-1}{2}-\nu\right)} \Gamma\left(\frac{d-1}{2}-\nu\right) \Gamma(\nu)}{4 \pi^{\frac{d d 1}{2}}} e^{-\left(\frac{d-1}{2}-\nu\right) s} \\
& +\frac{e^{-\mathrm{i} \pi\left(\frac{d-1}{2}+\nu\right)} \Gamma\left(\frac{d-1}{2}+\nu\right) \Gamma(-\nu)}{4 \pi^{\frac{d+1}{2}}} e^{-\left(\frac{d-1}{2}+\nu\right) s},
\end{aligned}
$$
}

which becomes oscillatory for the heavy mass case $m>(d-1) / 2$. The behavior for small $s$ is given by

$$
G^{+}\left(x(\tau), x(\tau-s) ; \eta_{0}\right) \sim \frac{e^{-\mathrm{i} \pi \frac{d-2}{2}} \Gamma\left(\frac{d-2}{2}\right)}{4 \pi^{\frac{d}{2}}}(s-\mathrm{i} 0)^{-(d-2)} .
$$

we give plots of the Wightman function for three typical cases of $d=4$ with $\nu=20 \mathrm{i}$ [heavy mass case; $m>(d-1) / 2=3 / 2$, Fig. 4], $\nu=0$ [ $m=3 / 2$, Fig. 5], and $\nu=1.4$ [light mass case; $m<3 / 2$, Fig. 6]. It is clear from these examples that, for the cases other than the light mass case (i.e., for the cases described in Figs. 4 and 5), the Wightman function $G^{+}\left(x(\tau), x(\tau-s) ; \eta_{0}\right)$ takes significant values only around the coincident point $s=0$. On the other hand, as can be seen in Fig. 6, the Wightman function has a longer-range correlation as the mass $m$ decreases. Thus, in the light mass case, we may not be able to neglect memory effects, and the Markovian approximation used in (30) may not be valid.

\section{APPENDIX E: CALCULATION OF $\dot{\mathcal{F}}$}

In this Appendix, we calculate the derivative of the response function

$$
\begin{aligned}
\dot{\mathcal{F}}_{a, b}^{(\alpha)}\left(\Delta E, \tau, \tau_{1}\right) \equiv & \int_{-\left(\tau-\tau_{1}\right)}^{0} \mathrm{~d} s e^{-\mathrm{i} \Delta E s} G_{a, b}^{(\alpha)}\left(x(\tau+s), x(\tau) ; \eta_{0}\right) \\
& +\int_{0}^{\left(\tau-\tau_{1}\right)} \mathrm{d} s e^{-\mathrm{i} \Delta E s} G_{a, b}^{(\alpha)}\left(x(\tau), x(\tau-s) ; \eta_{0}\right)
\end{aligned}
$$




\section{MASAFUMI FUKUMA, SOTARO SUGISHITA, AND YUHO SAKATANI}
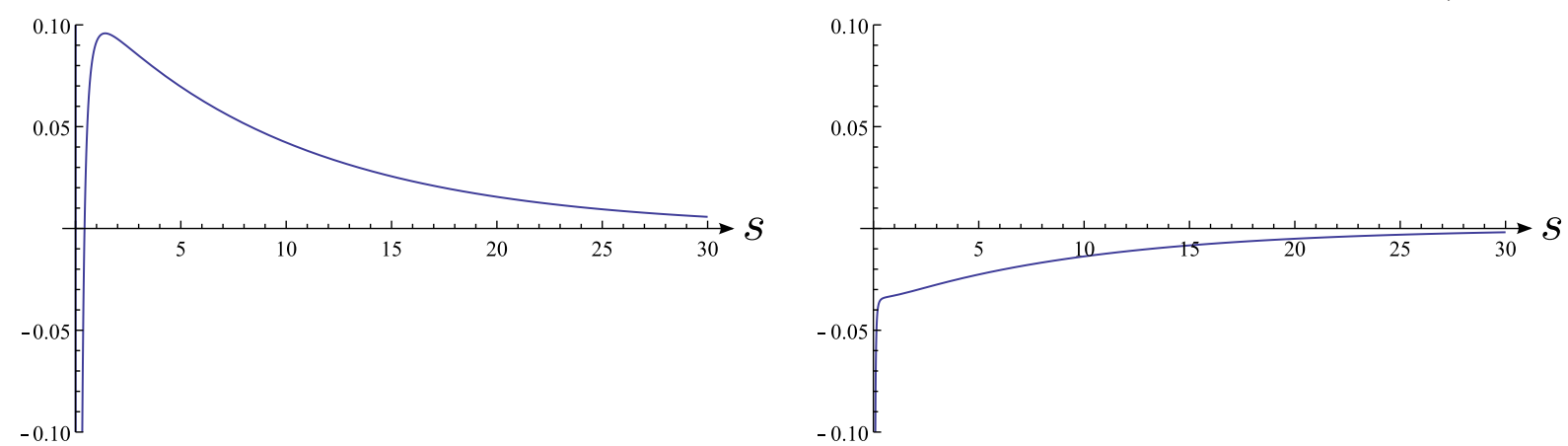

FIG. 6 (color online). The real part (left) and the imaginary part (right) of the Wightman function $G^{+}\left(x(\tau), x(\tau-s) ; \eta_{0}\right)$ for $d=4$, $\nu=1.4$ (light mass case), and $\tau-\tau_{0}=50$.

associated with the Wightman function [on the trajectory (40)] of the form

$$
\begin{aligned}
& G_{a, b}^{(\alpha)}\left(x, x^{\prime}\right) \\
& \quad=\frac{2\left[(-\eta)\left(-\eta^{\prime}\right)\right]^{\frac{d-1}{2}}}{(4 \pi)^{\frac{d-1}{2}} \Gamma\left(\frac{d-1}{2}\right)} \int_{0}^{\infty} \mathrm{d} k k^{d-2-\alpha} H_{\nu}^{(a)}(-k \eta) H_{\nu}^{(b)}\left(-k \eta^{\prime}\right) .
\end{aligned}
$$

Recall that $\dot{\mathcal{F}}$ associated with the Wightman function (43) is given by a linear combination of $\dot{\mathcal{F}}_{a, b}^{(\alpha)}$.

The first term in (E1) is rewritten to

$$
\begin{aligned}
& \int_{-\left(\tau-\tau_{1}\right)}^{0} \mathrm{~d} s e^{-\mathrm{i} \Delta E s} G_{a, b}^{(\alpha)}(x(\tau+s), x(\tau)) \\
& =\frac{2 e^{-\alpha \tau}}{(4 \pi)^{\frac{d-1}{2}} \Gamma\left(\frac{d-1}{2}\right)} \int_{0}^{\infty} d \mathbf{x} \mathbf{x}^{\frac{d-3}{2}+\mathrm{i} \Delta E} H_{\nu}^{(a)}\left(e^{\mathrm{i} \sigma_{a} \varepsilon} \mathbf{x}\right) \\
& \times \int_{e^{-\left(\tau-\tau_{1}\right)} \mathrm{x}}^{\mathrm{x}} d \mathrm{y} \mathrm{y}^{\frac{d-3}{2}-\alpha-\mathrm{i} \Delta E} H_{\nu}^{(b)}\left(e^{\mathrm{i} \sigma_{b} \varepsilon} \mathbf{y}\right) .
\end{aligned}
$$

Here, we have defined new variables $\mathrm{x} \equiv k e^{-(\tau+s)}$ and $\mathrm{y} \equiv k e^{-\tau}$, and the order of integration has been changed. In a similar way, the second term in (E1) is rewritten to

$$
\begin{aligned}
& \int_{0}^{\tau-\tau_{1}} \mathrm{~d} s e^{-\mathrm{i} \Delta E s} G_{a, b}^{(\alpha)}(x(\tau), x(\tau-s)) \\
& =\frac{2 e^{-\alpha \tau}}{(4 \pi)^{\frac{d-1}{2}} \Gamma\left(\frac{d-1}{2}\right)} \int_{0}^{\infty} \mathrm{dx} x^{\frac{d-3}{2}-\mathrm{i} \Delta E} H_{\nu}^{(b)}\left(e^{\mathrm{i} \sigma_{b} \varepsilon} \mathbf{x}\right) \\
& \quad \times \int_{e^{-\left(\tau-\tau_{1}\right)} \mathrm{x}}^{\mathrm{x}} \mathrm{dy} \mathrm{y}^{\frac{d-3}{2}-\alpha+\mathrm{i} \Delta E} H_{\nu}^{(a)}\left(e^{\mathrm{i} \sigma_{a} \varepsilon} \mathbf{y}\right),
\end{aligned}
$$

where we have defined $\mathbf{x} \equiv k e^{-(\tau-s)}$ and $\mathrm{y} \equiv k e^{-\tau}$ and again the order of integration has been changed.

The $y$ integration in (E3) and (E4) can be performed by using the following formula with $p=1$ and $q=e^{-\left(\tau-\tau_{1}\right)}$ :

$$
\begin{aligned}
& \int_{q \mathrm{x}}^{p \mathrm{x}} \mathrm{dy} \mathrm{y}^{\frac{d-3}{2}-\alpha \mp \mathrm{i} \Delta E} H_{\nu}^{(b)}\left(e^{\mathrm{i} \sigma_{b} \varepsilon} \mathrm{y}\right) \\
& =-\frac{2 \mathrm{i} \sigma_{b}}{\pi} e^{-\sigma_{b} \frac{\mathrm{i} \pi \nu}{2}} \int_{q \mathrm{x}}^{p \mathrm{x}} \mathrm{dy} \mathrm{y}^{\frac{d-3}{2}-\alpha \mp \mathrm{i} \Delta E} K_{\nu}\left(e^{-\sigma_{b} \frac{\mathrm{i}(\pi-2 \varepsilon)}{2}} \mathrm{y}\right) \\
& =-\frac{\mathrm{i} \sigma_{b}}{\pi} e^{-\sigma_{b} \frac{\mathrm{i} \pi \nu}{2}}\left[\int_{0}^{(p \mathrm{x})^{2}}-\int_{0}^{(q \mathrm{x})^{2}}\right] \mathrm{d} Y Y^{\frac{\frac{d-5}{2}-\alpha \mp \mathrm{i} \Delta E}{2}} \\
& \times K_{\nu}\left(e^{-\sigma_{b} \frac{\mathrm{i}(\pi-2 e)}{2}} \sqrt{Y}\right) \quad(\mathrm{y} \equiv \sqrt{Y}) \\
& =-\frac{\mathrm{i} \sigma_{b}}{\pi} e^{-\sigma_{b} \frac{\mathrm{i} \pi \nu}{2}} \int_{0}^{1} \mathrm{~d} \tilde{Y}\left[(p \mathbf{x})^{\frac{d-1}{2}-\alpha \mp \mathrm{i} \Delta E} \tilde{Y}^{\frac{d-5}{2}-\alpha \mp \mathrm{i} \Delta E}\right. \\
& \left.\times K_{\nu}\left(e^{-\sigma_{b} \frac{\mathrm{i}(\pi-2 \varepsilon)}{2}} a \times \sqrt{\tilde{Y}}\right)-(p \rightarrow q)\right] \\
& =-\frac{2^{\nu-1} \mathrm{i} \sigma_{b}}{\pi}\left[(p \mathbf{x})^{\frac{d-1}{2}-\nu-\alpha \mp \mathrm{i} \Delta E}\right. \\
& \left.\times G_{1,3}^{2,1}\left(\frac{-e^{2 \mathrm{i} \sigma_{b} \varepsilon}(a \mathbf{x})^{2}}{4} \mid \begin{array}{l}
\frac{\nu-\frac{d-5}{2}+\alpha \pm \mathrm{i} \Delta E}{2} \\
\nu, 0, \frac{\nu-\frac{d-1}{2}+\alpha \pm \mathrm{i} \Delta E}{2}
\end{array}\right)-(p \rightarrow q)\right] .
\end{aligned}
$$

In the last equality, we have used the following formula [see Eq. (6.592-2) of [21]]:

$$
\begin{gathered}
\int_{0}^{1} \mathrm{~d} x x^{\lambda} K_{\nu}(a \sqrt{x})=2^{\nu-1} a^{-\nu} G_{1,3}^{2,1}\left(\frac{a^{2}}{4} \mid \begin{array}{c}
\frac{\nu}{2}-\lambda \\
\nu, 0, \frac{\nu}{2}-\lambda-1
\end{array}\right) \\
{\left[\operatorname{Re} \lambda>-1+\frac{|\operatorname{Re} \nu|}{2}\right] .}
\end{gathered}
$$

If $q \neq 0$, the condition $\operatorname{Re} \lambda>-1+(|\operatorname{Re} \nu| / 2)[\Leftrightarrow(d-$ $1) / 2>|\operatorname{Re} \nu|+\alpha]$ is not necessary.

In order to perform the $\mathrm{x}$ integral in (E3) and (E4), we use 
MASTER EQUATION FOR THE UNRUH-DEWITT DETECTOR ...

PHYSICAL REVIEW D 89, 064024 (2014)

$$
\begin{aligned}
& \int_{0}^{\infty} \mathrm{d} x x^{\alpha-1} H_{\nu}^{(a)}(x) G_{1,3}^{2,1}\left(\frac{\omega x^{2}}{4} \mid \begin{array}{c}
a_{1} \\
b_{1}, b_{2}, b_{3}
\end{array}\right) \\
& =2^{\alpha-1}\left[G_{3,3}^{2,2}\left(\omega \mid \begin{array}{c}
\left.-\frac{\alpha+\nu-2}{2}, a_{1},-\frac{\alpha-\nu-2}{2}\right) \\
b_{1}, b_{2}, b_{3}
\end{array}\right)\right. \\
& \left.\quad+\mathrm{i} \sigma_{a} G_{4,4}^{2,3}\left(\omega \mid \begin{array}{c}
-\frac{\alpha+\nu-2}{2},-\frac{\alpha-\nu-2}{2}, a_{1},-\frac{\alpha-\nu-3}{2} \\
b_{1}, b_{2}, b_{3},-\frac{\alpha-\nu-3}{2}
\end{array}\right)\right],
\end{aligned}
$$

which can be derived from formulas 7.821-1 and 7.821-2 of [21]:

$$
\begin{aligned}
& \int_{0}^{\infty} \mathrm{d} x x^{\alpha-1} J_{\nu}(x) G_{1,3}^{2,1}\left(\frac{\omega x^{2}}{4} \mid \begin{array}{c}
a_{1} \\
b_{1}, b_{2}, b_{3}
\end{array}\right) \\
& =2^{\alpha-1} G_{3,3}^{2,2}\left(\omega \mid \begin{array}{c}
-\frac{\alpha+\nu-2}{2}, a_{1},-\frac{\alpha-\nu-2}{2} \\
b_{1}, b_{2}, b_{3}
\end{array}\right), \\
& \int_{0}^{\infty} \mathrm{d} x x^{\alpha-1} N_{\nu}(x) G_{1,3}^{2,1}\left(\frac{\omega x^{2}}{4} \mid \begin{array}{c}
a_{1} \\
b_{1}, b_{2}, b_{3}
\end{array}\right) \\
& =2^{\alpha-1} G_{4,4}^{2,3}\left(\omega \mid \begin{array}{c}
-\frac{\alpha+\nu-2}{2},-\frac{\alpha-\nu-2}{2}, a_{1},-\frac{\alpha-\nu-3}{2} \\
b_{1}, b_{2}, b_{3},-\frac{\alpha-\nu-3}{2}
\end{array}\right) .
\end{aligned}
$$

$$
\begin{aligned}
& \frac{2 e^{-\alpha \tau}}{(4 \pi)^{\frac{d-1}{2}} \Gamma\left(\frac{d-1}{2}\right)} \int_{0}^{\infty} \mathrm{dx} x^{\frac{d-3}{2} \pm \mathrm{i} \Delta E} H_{\nu}^{(a)}\left(e^{\mathrm{i} \sigma_{a} \varepsilon} \mathbf{x}\right) \int_{e^{-\left(\tau-\tau_{1}\right) \mathrm{x}}}^{\mathrm{x}} \mathrm{dy} \mathrm{y}^{\frac{d-3}{2}-\alpha \mp \mathrm{i} \Delta E} H_{\nu}^{(b)}\left(e^{\mathrm{i} \sigma_{b} \varepsilon} \mathbf{y}\right) \\
& =-\frac{i \sigma_{b} e^{-\alpha \tau}}{2^{\alpha+1} \pi^{\frac{d+1}{2}} \Gamma\left(\frac{d-1}{2}\right)} \times\left[G_{3,3}^{2,2}\left(-e^{-2 \mathrm{i}\left(\sigma_{a}-\sigma_{b}\right) \varepsilon} \mid \begin{array}{c}
-\frac{d-3-\alpha}{2}, \frac{\nu-\frac{d-5}{2}+\alpha \pm \mathrm{i} \Delta E}{2},-\frac{d-3-\alpha}{2}+\nu \\
\nu, 0, \frac{\nu \frac{d-1}{2}+\alpha \pm \mathrm{i} \Delta E}{2}
\end{array}\right)\right. \\
& +\mathrm{i} \sigma_{a} G_{4,4}^{2,3}\left(-e^{-2 \mathrm{i}\left(\sigma_{a}-\sigma_{b}\right) \varepsilon} \mid \begin{array}{c}
-\frac{d-3-\alpha}{2},-\frac{d-3-\alpha}{2}+\nu, \frac{\nu-\frac{d-5}{2}+\alpha \pm \mathrm{i} \Delta E}{2},-\frac{d-4-\alpha}{2}+\nu \\
\nu, 0, \frac{\nu-\frac{d-1}{2}+\alpha \pm \mathrm{i} \Delta E}{2},-\frac{d-4-\alpha}{2}+\nu
\end{array}\right)
\end{aligned}
$$

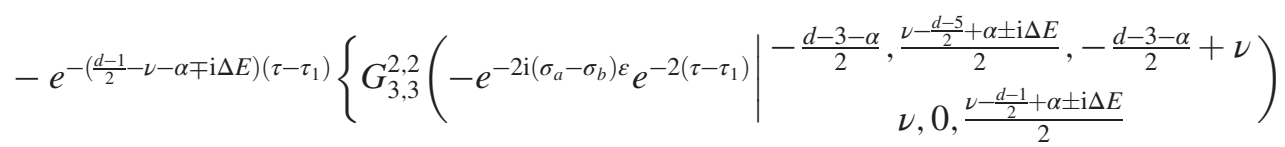

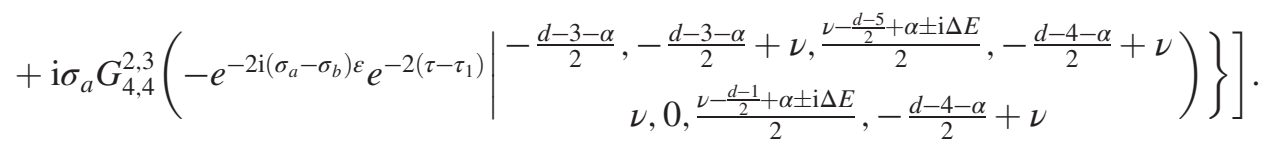

Combining (E1), (E3), (E4), (E10), and (B10), we finally obtain the following expression for $\dot{\mathcal{F}}_{a, b}^{(\alpha)}\left(\Delta E, \tau, \tau_{1}\right)$ :

$$
\begin{aligned}
\dot{\mathcal{F}}_{a, b}^{(\alpha)}\left(\Delta E, \tau, \tau_{1}\right)= & b_{a, b}^{(\alpha, 0)} e^{-\alpha \tau}+b_{a, b}^{(\alpha, 1)} e^{-\left(\frac{d-1}{2}+\nu-\mathrm{i} \Delta E\right) \tau} \\
& +b_{a, b}^{(\alpha, 2)} e^{-\left(\frac{d-1}{2}-\nu-\mathrm{i} \Delta E\right) \tau} \\
& +b_{a, b}^{(\alpha, 3)} e^{-\left(\frac{d-1}{2}+\nu+\mathrm{i} \Delta E\right) \tau} \\
& +b_{a, b}^{(\alpha, 4)} e^{-\left(\frac{d-1}{2}-\nu+\mathrm{i} \Delta E\right) \tau}
\end{aligned}
$$

where

$$
\begin{aligned}
b_{a, b}^{(\alpha, 0)} \equiv & \left(-e^{-\left(\sigma_{a}-\sigma_{b}\right) \mathrm{i} 0}\right)^{\nu} \mathbf{F}_{a, b}^{(\alpha)}(\nu, \Delta E ; 1) \\
& +e^{-\sigma_{a} \mathrm{i} \pi \nu} \mathbf{F}_{a, b}^{(\alpha)}(-\nu, \Delta E ; 1) \\
& +\left(-e^{\left(\sigma_{a}-\sigma_{b}\right) \mathrm{i} 0}\right)^{\nu} \mathbf{F}_{b, a}^{(\alpha)}(\nu,-\Delta E ; 1) \\
& +e^{-\sigma_{b} \mathrm{i} \pi \nu} \mathbf{F}_{b, a}^{(\alpha)}(-\nu,-\Delta E ; 1)
\end{aligned}
$$

$$
b_{a, b}^{(\alpha, 2)} \equiv-e^{-\sigma_{a} \mathrm{i} \pi \nu} e^{\left(\frac{d-1}{2}-\nu-\alpha-\mathrm{i} \Delta E\right) \tau_{1}} \mathbf{F}_{a, b}^{(\alpha)}\left(-\nu, \Delta E ; e^{-2\left(\tau-\tau_{1}\right)}\right),
$$

$$
\begin{aligned}
b_{a, b}^{(\alpha, 1)} \equiv & -\left(-e^{-\left(\sigma_{a}-\sigma_{b}\right) \mathrm{i} 0}\right)^{\nu} e^{\left(\frac{d-1}{2}+\nu-\alpha-\mathrm{i} \Delta E\right) \tau_{1}} \\
& \times \mathbf{F}_{a, b}^{(\alpha)}\left(\nu, \Delta E ; e^{-2\left(\tau-\tau_{1}\right)}\right)
\end{aligned}
$$

$$
b_{a, b}^{(\alpha, 4)} \equiv-e^{-b \mathrm{i} \pi \nu} e^{\left(\frac{d-1}{2}-\nu-\alpha+\mathrm{i} \Delta E\right) \tau_{1}} \mathbf{F}_{b, a}^{(\alpha)}\left(-\nu,-\Delta E ; e^{-2\left(\tau-\tau_{1}\right)}\right),
$$




$$
\begin{aligned}
\mathbf{F}_{a, b}^{(\alpha)}(\nu, \Delta E ; x) & \\
\equiv & \frac{e^{\sigma_{a} \mathrm{i} \pi \frac{d-\alpha}{2}} e^{-\sigma_{b}{ }^{\frac{\pi}{2}}}}{2^{\alpha+1} \pi^{\frac{d+1}{2}} \Gamma\left(\frac{d-1}{2}\right) \sin (\pi \nu)} \\
& \times{ }_{3} \hat{F}_{2}\left(\begin{array}{c}
\frac{d-1-\alpha}{2}, \frac{d-1-\alpha}{2}+\nu, \frac{\frac{d-1}{2}+\nu-\alpha-\mathrm{i} \Delta E}{2} \\
1+\nu, \frac{\frac{d+3}{2}+\nu-\alpha-\mathrm{i} \Delta E}{2}
\end{array} e^{-\mathrm{i}\left(\sigma_{a}-\sigma_{b}\right) 0} x\right) .
\end{aligned}
$$

Since $b_{a, b}^{(\alpha, 0)}$ is independent of $\tau$, and $b_{a, b}^{(\alpha, i)}(i=1,2,3,4)$ become independent of $\tau$ in the limit $\tau \rightarrow \infty$, we find $\dot{\mathcal{F}}_{a, b}^{(\alpha)}$ to take the following asymptotic form at later times:

$$
\dot{\mathcal{F}}_{a, b}^{(\alpha)} \sim \text { const } e^{-\alpha \tau}+\text { const } e^{-\left(\frac{d-1}{2} \pm \nu \pm \mathrm{i} \Delta E\right)\left(\tau-\tau_{1}\right)} .
$$

Then, for the Wightman function $\Delta G_{k}^{+}\left(\eta, \eta^{\prime}\right)$ given in (49), the derivative of the response function becomes

$$
\begin{aligned}
\Delta \dot{\mathcal{F}}\left(\Delta E ; \tau, \tau_{1}\right) & \sim \sum_{a, b=1}^{2} \operatorname{const} \dot{\mathcal{F}}_{a, b}^{\left(\alpha_{a b}\right)} \\
& \sim \sum_{a, b=1}^{2}\left[\operatorname{const} e^{-\alpha_{a b} \tau}+\operatorname{const} e^{-\left(\frac{d-1}{2} \pm \nu \pm \mathrm{i} \Delta E\right)\left(\tau-\tau_{1}\right)}\right] \\
& \sim \text { const } e^{-\alpha \tau}+\text { const } e^{-\left(\frac{d-1}{2} \pm \nu \pm \mathrm{i} \Delta E\right)\left(\tau-\tau_{1}\right)} \quad(\mathrm{E} 19)
\end{aligned}
$$

with $\alpha \equiv \min _{a, b}\left(\alpha_{a b}\right)$.

The derivative of the response function for the BunchDavies vacuum, $\dot{\mathcal{F}}_{\mathrm{BD}}$, can also be calculated from (E11) by setting $a=1, b=2$, and $\alpha=0$ :

$$
\begin{aligned}
\dot{\mathcal{F}}_{\mathrm{BD}}\left(\Delta E, \tau, \tau_{1}\right)= & \frac{\pi}{4} \dot{\mathcal{F}}_{1,2}^{(0)}\left(\Delta E, \tau, \tau_{1}\right) \\
= & \dot{\mathcal{F}}^{\mathrm{eq}}(\Delta E)+\frac{\pi}{4} b_{1,2}^{(0,1)} e^{-\left(\frac{d-1}{2}+\nu-\mathrm{i} \Delta E\right) \tau} \\
& +\frac{\pi}{4} b_{1,2}^{(0,2)} e^{-\left(\frac{d-1}{2}-\nu-\mathrm{i} \Delta E\right) \tau} \\
& +\frac{\pi}{4} b_{1,2}^{(0,3)} e^{-\left(\frac{d-1}{2}+\nu+\mathrm{i} \Delta E\right) \tau} \\
& +\frac{\pi}{4} b_{1,2}^{(0,4)} e^{-\left(\frac{d-1}{2}-\nu+\mathrm{i} \Delta E\right) \tau}
\end{aligned}
$$

where

$$
\begin{aligned}
& \dot{\mathcal{F}}^{\mathrm{eq}}(\Delta E) \\
& \equiv \frac{\pi}{4} b_{1,2}^{(0,0)} \\
& =\frac{e^{-\pi \Delta E} \Gamma\left(\frac{\frac{d-1}{2}+\nu+\mathrm{i} \Delta E}{2}\right) \Gamma\left(\frac{\frac{d-1}{2}-\nu+\mathrm{i} \Delta E}{2}\right) \Gamma\left(\frac{\frac{d-1}{2}+\nu-\mathrm{i} \Delta E}{2}\right) \Gamma\left(\frac{\frac{d-1}{2}-\nu-\mathrm{i} \Delta E}{2}\right)}{8 \pi^{\frac{d+1}{2}} \Gamma\left(\frac{d-1}{2}\right)},
\end{aligned}
$$

$$
b_{1,2}^{(0,1)}=-e^{\mathrm{i} \pi \nu} e^{\left(\frac{d-1}{2}+\nu-\mathrm{i} \Delta E\right) \tau_{1}} \mathbf{F}_{1,2}^{(0)}\left(\nu, \Delta E ; e^{-2\left(\tau-\tau_{1}\right)}\right),
$$

$$
b_{1,2}^{(0,2)}=-e^{-\mathrm{i} \pi \nu} e^{\left(\frac{d-1}{2} \nu-\mathrm{i} \Delta E\right) \tau_{1}} \mathbf{F}_{1,2}^{(0)}\left(-\nu, \Delta E ; e^{-2\left(\tau-\tau_{1}\right)}\right),
$$

$$
b_{1,2}^{(0,3)}=-e^{-\mathrm{i} \pi \nu} e^{\left(\frac{d-1}{2}+\nu+\mathrm{i} \Delta E\right) \tau_{1}} \mathbf{F}_{2,1}^{(0)}\left(\nu,-\Delta E ; e^{-2\left(\tau-\tau_{1}\right)}\right),
$$

$$
b_{1,2}^{(0,4)}=-e^{\mathrm{i} \pi \nu} e^{\left(\frac{d-1}{2}-\nu+\mathrm{i} \Delta E\right) \tau_{1}} \mathbf{F}_{2,1}^{(0)}\left(-\nu,-\Delta E ; e^{-2\left(\tau-\tau_{1}\right)}\right) .
$$

We have used (B14) to obtain (E21). Equations (E20)(E25) give Eq. (59), which shows that $\dot{\mathcal{F}}_{\mathrm{BD}}\left(\Delta E, \tau, \tau_{1}\right)$ has the following asymptotic form for $\tau \rightarrow \infty$ :

$$
\dot{\mathcal{F}}_{\mathrm{BD}}\left(\Delta E, \tau, \tau_{1}\right) \sim \dot{\mathcal{F}}^{\mathrm{eq}}(\Delta E)+\text { const } e^{-\left(\frac{d-1}{2} \pm \nu \pm \mathrm{i} \Delta E\right) \tau} .
$$

[1] W. G. Unruh, Phys. Rev. D 14, 870 (1976).

[2] B. S. DeWitt in General Relativity: An Einstein Centenary Survey, edited by S. W. Hawking and W. Israel (Cambridge University Press, Cambridge, England, 1979).

[3] N. D. Birrell and P.C.W. Davies, Quantum Fields in Curved Space (Cambridge University Press, Cambridge, England, 1982).

[4] S. Takagi, Prog. Theor. Phys. Suppl. 88, 1 (1986).
[5] E. Mottola, Phys. Rev. D 31, 754 (1985).

[6] B. Allen, Phys. Rev. D 32, 3136 (1985).

[7] T. S. Bunch and P. C. W. Davies, Proc. R. Soc. A 360, 117 (1978).

[8] M. Spradlin, A. Strominger, and A. Volovich, arXiv:hep-th/ 0110007.

[9] R. Bousso, A. Maloney, and A. Strominger, Phys. Rev. D 65, 104039 (2002). 


\section{MASTER EQUATION FOR THE UNRUH-DEWITT DETECTOR ...}

PHYSICAL REVIEW D 89, 064024 (2014)

[10] R. Figari, R. Hoegh-Krohn, and C. R. Nappi, Commun. Math. Phys. 44, 265 (1975).

[11] G. W. Gibbons and S. W. Hawking, Phys. Rev. D 15, 2738 (1977).

[12] M. Fukuma, Y. Sakatani, and S. Sugishita, Phys. Rev. D 88, 024041 (2013).

[13] R. Kubo, M. Toda, and N. Hashitsume, Statistical Physics II (Springer, Berlin, 1985).

[14] B. Garbrecht and T. Prokopec, Classical Quantum Gravity 21, 4993 (2004).

[15] A. Higuchi, Classical Quantum Gravity 4, 721 (1987).

[16] L. Parker, Ph.D. thesis, Harvard University, 1966.

[17] L. Parker and S. A. Fulling, Phys. Rev. D 9, 341 (1974).

[18] L. E. Parker and D. J. Toms, Quantum Field Theory in Curved Spacetime: Quantized Field and Gravity (Cambridge University Press, Cambridge, England, 2009).

[19] P. R. Anderson, W. Eaker, S. Habib, C. Molina-Paris, and E. Mottola, Phys. Rev. D 62, 124019 (2000).
[20] P. R. Anderson, C. Molina-Paris, and E. Mottola, Phys. Rev. D 72, 043515 (2005).

[21] I. S. Gradshteyn and I. M. Ryzhik, in Table of Integrals, Series, and Products, edited by Alan Jeffrey and Daniel Zwillinger (Academic Press, New York, 2007), 7th ed.

[22] The Wolfram Functions Site, http://functions.wolfram.com/ 07.34.26.0004.01.

[23] The Wolfram Functions Site, http://functions.wolfram.com/ 07.27.17.0026.01.

[24] S. Schlicht, Classical Quantum Gravity 21, 4647 (2004).

[25] P. Langlois, Ann. Phys. (Amsterdam) 321, 2027 (2006).

[26] A. Satz, Classical Quantum Gravity 24, 1719 (2007).

[27] J. Louko and A. Satz, Classical Quantum Gravity 25, 055012 (2008).

[28] L. Hodgkinson and J. Louko, J. Math. Phys. (N.Y.) 53, 082301 (2012).

[29] I. Trigui, S. Affes, and A. Stephenne, in Proceedings of the Global Telecommunications Conference (GLOBECOM 2010) (IEEE, New York, 2010), pp. 1-5. 